Anuario Latinoamericano Ciencias Políticas

y Relaciones Internacionales

vol. 7, 2019

pp. $75-109$

\section{La Alianza del Pacífico a ocho años de su creación. Balance crítico y perspectivas}

\section{The Pacific Alliance Eight Years after Its Creation. Critical Balance and Perspectives}

\author{
Geneviève Marchini* \\ DEPARTAMENTO DE ESTUDIOS DEL PACÍFICO \\ UNIVERSIDAD DE GUADALAJARA \\ MÉXICO \\ $\triangle$ genevievemarchini@gmail.com \\ https://orcid.org/0000-0002-3730-2235
}

\title{
RESUMEN
}

A ocho años de la Declaración de Lima (28 de abril de 2011), la Alianza del Pacífico sigue suscitando interés internacional. Este mecanismo de integración surgió en un contexto de transición de la economía global hacia un mundo multipolar, en el cual Asia del Este, liderada por la República Popular China y Japón, ha adquirido un mayor protagonismo económico. En este contexto, la Alianza se creó como un mecanismo de integración regional novedoso, que estableció un doble objetivo: integración entre sus miembros y proyección al mundo, en especial hacia el AsiaPacífico.

Este texto se propone analizar y caracterizar la Alianza del Pacífico, e interrogarse acerca del potencial de este mecanismo de integración para: 1. cumplir con el doble objetivo que se propone, creando sinergias entre los distintos aspectos que lo integran; $y$ 2. transformarse en una plataforma de cooperación y en un nuevo modelo de integración para América Latina. Para ello, resume en la primera sección los hitos más importantes de la Alianza y sus principales iniciativas. En la segunda sección, aborda el debate relativo a sus objetivos y caracterización en tanto mecanismo de integración regional. La tercera sección se dedica a las expectativas que generó la Alianza en materia de integración intrarregional y extrarregional y a las condiciones necesarias para su éxito, mientras que la cuarta sección discute

\footnotetext{
* Profesora e investigadora del Departamento de Estudios del Pacífico de la Universidad de Guadalajara (Centro Universitario de Ciencias Sociales y Humanidades) e investigadora nacional nivel II (Conacyt, México). Es Doctora en Economía por la Universidad de Paris XIII (Francia). Sus temas de investigación recientes versan sobre relaciones económicas Alianza del Pacífico-Asia del Este, desarrollo económico y financiero en Asia del Este y en América Latina, cooperación financiera regional, criptomonedas y regulación en Asia del Este.
} 
Dossier América Latina: continuidad y cambio en el escenario regional las perspectivas de este mecanismo a la luz de acontecimientos y cambios recientes en la economía global.

PALABRAS CLAVE: Alianza del Pacífico, integración regional, relaciones económicas interregionales, América Latina, Asía-Pacífico.

\section{ABSTRACT}

Eight years after the Declaration of Lima (April 28, 2011), the Pacific Alliance continues to generate international interest. This integration mechanism emerged in a context of transition of the global economy to a multipolar world, in which East Asia, led by the People's Republic of China and Japan, has acquired a greater economic role. In these circumstances, the Alliance was created as a new regional integration mechanism, which pursues a double objective: integration among its members and projection to the world, especially towards Asia-Pacific.

This text seeks to analyze and characterize the Pacific Alliance, and to question the potential of this integration mechanism as to: 1. fulfilling its double objective by creating synergies between the different aspects that integrate it and, 2. becoming a cooperation platform and a new integration model for Latin America. For this, in the first section, it summarizes the most important milestones of the Alliance and its main initiatives. In the second section, it addresses the debate regarding its objectives and characterization as a regional integration mechanism. The third section is devoted to the expectations generated by the Alliance in terms of intraregional and extraregional integration and the conditions necessary for its success, while the fourth section discusses the perspectives of this mechanism in light of recent events and changes in the global economy.

KEYWORDS: Pacific Alliance, regional integration, interregional economic relations, Latin America, Asia-Pacific.

\section{Introducción}

A ocho años de la Declaración de Lima (28 de abril de 2011), la Alianza del Pacífico (AP) sigue suscitando interés internacional. En el plano de las relaciones internacionales, éste se observa en primer lugar en el creciente número de países observadores del mecanismo: 55 en febrero de 2019'1, originarios de todos los continentes, incluyendo 14 países de las Américas, 28 países europeos, 9 observadores de Asia, 2 de África y 2 de Oceanía ${ }^{2}$. En segundo lugar, el in-

\footnotetext{
1 Información extraída el 13 de febrero de 2019 de https://alianzapacifico.net/paises observadores/. En mayo, se acordó el ingreso de Kazajistán y Azerbaiyán, (Secretaría de Relaciones Exteriores de México, mayo 2019).

2 A título de comparación, la Comunidad Andina de Naciones (CAN), a la cual pertenecen tanto Colombia como Perú, tiene cinco países asociados -los cinco países del Cono Sur de América Latina, incluyendo Chile- y un único país observador, España, http://www.comunidadandina.org.
} 
terés se manifiesta en el apoyo técnico brindado por organismos internacionales, en particular el BID (Banco Interamericano de Desarrollo), la CEPAL (Comisión Económica para América Latina) o la OCDE (Organización para la Cooperación y el Desarrollo Económico). En tercer lugar, siguen enriqueciéndose las publicaciones dedicadas a la AP: después de la prensa y los think tanks internacionales, la comunidad académica regional e internacional se ha abocado a investigar numerosos aspectos de la Alianza desde distintos ángulos teóricos y disciplinarios, en especial desde la economía política internacional y desde la economía. Estas contribuciones se pueden ordenar en distintos ejes: los objetivos y las características institucionales de la Alianza constituyen un primer eje de discusión, el cual lleva a un segundo eje que debate los resultados esperados de su puesta en práctica y su impacto a nivel regional.

La Alianza del Pacífico (AP) surgió en un contexto de transición de la economía global hacia un mundo multipolar, en el cual Asia del Este, liderada por la República Popular China (China, de aquí en adelante) y Japón, ha adquirido un mayor protagonismo económico. Éste se manifestó en América Latina a través de un alza rápida de los intercambios comerciales, en ciertos casos asociada a una creciente presencia de inversionistas originarios de esta región del mundo. En este contexto, la Alianza se creó como un mecanismo (Comunidad Andina desde 1996, anteriormente Pacto Andino, 1969) de integración regional novedoso, que estableció un doble objetivo: integración entre sus miembros y proyección al mundo, en especial hacia el Asia-Pacífico. La AP presenta varias particularidades que la diferencian de los mecanismos anteriores de la integración latinoamericana, si bien, como lo veremos, recoge muchos de sus principales objetivos. Así, se fundamenta no tanto en una cercanía geográfica, sino en una visión económica y política común, en la cual destacan la ortodoxia macroeconómica y la búsqueda de una inserción internacional basada en el libre comercio. La “integración profunda”, que busca promover entre sus miembros, acompañada de iniciativas de cooperación en distintos ámbitos, difiere también de las etapas clásicas de la integración regional teorizadas en las décadas anteriores. Finalmente, otra diferencia sobresaliente se destaca en la forma en que la AP se propone alcanzar sus objetivos: a través de mecanismos estrictamente intergubernamentales, sin creación de instituciones supranacionales, en contraste con un mecanismo regional de integración como la CAN (Comunidad Andina de Naciones), a la cual pertenecen Colombia y Perú.

Este artículo se propone analizar y caracterizar la Alianza del Pacífico, e interrogarse acerca del potencial de este mecanismo de integración para cumplir con el doble objetivo que se propone, creando sinergias entre los distintos aspectos que lo conforman, y transformarse en una plataforma de cooperación y en un nuevo modelo de integración para América La-

El MERCOSUR (Mercado Común del Sur) no contempla la figura de Estado observador y, en abril de 2019, tenía siete países asociados, entre los cuales se encuentran Chile, Colombia y Perú. https://www.mercosur.int/quienes-somos/paises-del-mercosur/.
La Alianza del Pacífico a ocho años de su creación. Balance crítico y perspectivas Geneviève Marchini 


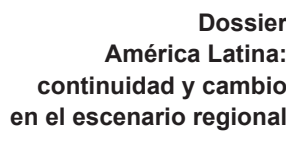

Dossier

tina. Para ello, resume en una primera sección los hitos más importantes de la Alianza y sus principales iniciativas. En la segunda sección, el artículo aborda el debate relativo a sus objetivos y a su caracterización en tanto mecanismo de integración regional. La tercera sección se dedica a las expectativas que generó la AP en materia de integración intrarregional y extrarregional y a las condiciones necesarias para su éxito, mientras que la cuarta sección discute las perspectivas de este mecanismo a la luz de acontecimientos y cambios recientes en la economía global.

\section{La Alianza del Pacífico: principales hitos}

El 28 de abril de 2011, la Declaración de Lima propuso “...la Alianza del Pacífico como un área de integración profunda [...] que aliente la integración regional, así como un mayor crecimiento, desarrollo y competitividad de nuestras economías" y afirmó el compromiso de "avanzar progresivamente hacia la libre circulación de bienes, servicios, capitales y personas”. A la vez, se planteaba que ello permitiría proyectar los países miembros "con mayor competitividad, especialmente hacia la región del Asia-Pacífico" (Alianza del Pacífico 2011a: 1-2). La declaración se cerraba con una invitación a participar dirigida a los países de la región "que compartan la voluntad de alcanzar las metas de esta Alianza" (Alianza del Pacífico 2011a: 1-2).

El Acuerdo Marco, firmado un año después, en la IV Cumbre de Paranal (abril de 2012), estableció la Alianza del Pacífico como "área de integración regional" (art. 1), y estipuló en el art. 2 los requisitos de vigencia del Estado de derecho, democracia, separación de poderes y respeto de los derechos humanos, para todo país que quisiera postular al mecanismo. Planteó luego como objetivos de la Alianza:

a. construir, de manera participativa y consensuada, un área de integración profunda para avanzar progresivamente hacia la libre circulación de bienes, servicios, capitales y personas;

b. impulsar un mayor crecimiento, desarrollo y competitividad de las economías de las Partes, con miras a lograr un mayor bienestar, la superación de la desigualdad socioeconómica y la inclusión social de sus habitantes; $y$

c. convertirse en una plataforma de articulación política, de integración económica y comercial, y de proyección al mundo, con especial énfasis al Asia-Pacífico (Alianza del Pacífico 2012).

Por otro lado, el art. 8 afirma que las decisiones y los acuerdos tomados en el marco de la Alianza "no reemplazarán, ni modificaran los acuerdos económicos, comerciales y de integración bilaterales, regionales, multilaterales vigentes entre las Partes", y el art. 11 establece que "El presente Acuerdo Marco 
quedará abierto a la adhesión de los Estados que así lo soliciten y tengan vigente un acuerdo de libre comercio con cada una de las Partes".

En el plano institucional, la Declaración de Lima estableció el Grupo de Alto Nivel (GAN) conformado por los viceministros de Relaciones Exteriores y de Comercio de los cuatro países, mientras que el Acuerdo Marco creó el Consejo de Ministros (art. 4), definió sus participantes -ministros de Relaciones Exteriores y de Comercio Exterior-, y sus atribuciones. Se estableció la obligación de sesionar con la presencia de todas las partes y de tomar decisiones por consenso (art. 5). En el art. 7, se establecieron las atribuciones de la Presidencia pro tempore y el principio de rotación anual entre los países miembros.

Simultáneamente, la Alianza del Pacífico ha buscado dotarse de una serie de mecanismos de cooperación, que se agregan a los objetivos de liberalización de flujos económicos y de "integración profunda”. En diciembre de 2011, en la segunda cumbre de mandatarios, se firmó el Memorando de Entendimiento sobre la Plataforma de Cooperación del Pacífico (2011), que incluyó entre sus metas iniciales la cooperación en cuatro áreas específicas: el medio ambiente y el cambio climático, la innovación, la ciencia y la tecnología, las Micro, Pequeñas y Medianas Empresas (MPyMEs) y el desarrollo social. En esta misma cumbre, se formalizó el apoyo de las autoridades de los cuatro países al Mercado Integrado Latinoamericano (MILA), una iniciativa privada de integración de las bolsas de valores de los tres países andinos en función desde mayo de 2011, a la cual se sumó la Bolsa Mexicana de Valores en 2014. Por otro lado, la Alianza ha sido dotada tempranamente de un organismo empresarial. El Consejo Empresarial de la Alianza del Pacífico (CEAP), establecido en noviembre de 2012, se ha propuesto temas de trabajo bastante concretos y precisos y se generó una dinámica entre éste y las Cumbres de mandatarios, a las cuales entrega recomendaciones. La AP creó también mecanismos de intercambio estudiantil y académico con ambiciones modestas pero activos casi desde sus inicios.

Estas notas dejan claro que los objetivos establecidos por la Alianza se centran alrededor de la apertura económica y la integración, concebidas como medios para fortalecer la competitividad de sus miembros y para alcanzar metas de crecimiento económico, desarrollo y equidad social. Queda también claro que la Alianza se define en parte por su ambición de proyectarse hacia el Asia-Pacífico y que las instituciones que plantea son exclusivamente de tipo intergubernamental, con decisiones que se deberán tomar por consenso y unanimidad en distintos niveles: Presidencia, Consejo de Ministros, Grupo de Alto Nivel, estos últimos integrados por funcionarios de cada país.

A la vez, los planteamientos iniciales de la AP muestran niveles de ambigüedad o de indefinición, que los avances realizados en cerca de ocho años de existencia han despejado sólo en parte. Éstos se relacionan en primer lugar con el contenido económico preciso que cabe atribuir al "área de integración profunda" que se pretende construir: ¿zona de libre-comercio, mercado
La Alianza del Pacífico a ocho años de su creación. Balance crítico y perspectivas Geneviève Marchini 
Dossier América Latina: continuidad y cambio en el escenario regional común, integración productiva entre los miembros y/o con socios externos? En segundo lugar, tienen que ver con el alcance geográfico del mecanismo, que La Declaración de Lima establecía inicialmente en la región latinoamericana, pero que pareció abrirse a miembros potenciales más allá de esta región en la redacción del Acuerdo Marco. Finalmente, ¿qué sentido dar en el ámbito regional latinoamericano al rol de plataforma de "articulación política, de integración económica y comercial, y de proyección al mundo"?

¿Cuáles han sido los ámbitos con mayores avances en la existencia de la Alianza? En forma preliminar, se puede afirmar que éstos son, sobre todo, la liberalización y facilitación de los flujos de bienes y servicios, los mercados de capitales, el relacionamiento externo y, en segundo lugar, los flujos de personas y algunas actividades de cooperación. En materia de liberalización de los flujos de bienes y servicios, sobresale la firma (febrero de 2014) y entrada en vigor (2016) del Protocolo Adicional del Acuerdo Marco, integrado por 19 capítulos que estipulan los acuerdos tomados en materia de liberalización del comercio de bienes y servicios, de inversión, de reglas de origen y de acumulación de origen -posible clave de un fortalecimiento de cadenas productivas regionales-, de facilitación comercial y cooperación aduanera, de contratación pública y de solución de diferencias. Cabe mencionar también logros posteriores en materia de facilitación de comercio, con los avances en la interoperabilidad de las Ventanillas Únicas de Comercio Exterior, el Reconocimiento Mutuo de Operador Económico Autorizado y en la armonización de normas (productos farmacéuticos, dispositivos médicos, cosméticos, suplementos alimenticios).

El objetivo de fortalecimiento de las cadenas productivas regionales, que no era explícito, se introdujo en 2014 y constituye un hito importante en la formulación de objetivos de integración regional: se menciona en la Cumbre de Cartagena en la que se firmó el Protocolo Adicional, al señalarse como beneficio esperado del protocolo "una mayor integración en cadenas regionales de valor que harán más competitiva a la región” y en junio de 2014, el anexo de la Declaración Presidencial de Punta de Mita estableció acciones para concretizar este objetivo. $^{3}$

El segundo ámbito que muestra adelantos importantes es el del mercado de capitales, cuya integración presenta numerosos retos regulatorios y tributarios, y del financiamiento del desarrollo. Sobresalen los avances para la operación regional de fondos de pensiones y fondos de inversión, la emisión conjunta de un bono regional catastrófico en los mercados globales ${ }^{4}$, la creación de un Fondo de Capital Emprendedor para MPyMEs y los progresos en la im-

3 El Anexo de la Declaración encargó varias tareas relacionadas a las agencias de promoción de los cuatro países: "establecer un modelo operativo que permita concretar encadenamientos productivos entre los estados miembros [...], generar [...] una estrategia de complementación para los sectores económicos priorizados por los países de la Alianza del Pacífico" y "promover las exportaciones de las micro y pequeñas empresas" (Alianza del Pacífico 2014b).

4 En 2018, la Alianza del Pacífico colocó un bono por 1,4 billones de US\$, el mayor en valor en aquel momento, patrocinado por el Banco Mundial (Ralph 2018). 
plementación del Fondo de Infraestructura Regional. Por otro lado, se dieron algunas medidas de liberalización en materia de flujos de personas, entre las cuales sobresalen la eliminación de visas para estancias temporales, y las negociaciones concluidas para el registro voluntario de ingenieros con miras al otorgamiento de licencias temporales para el ejercicio y la exportación de estos servicios en los países de la AP. También pueden incluirse en este rubro los intercambios estudiantiles y académicos.

El relacionamiento externo registra numerosas iniciativas nuevas importantes y el seguimiento de las metas en curso, una intensificación que denota los esfuerzos de adaptación de la Alianza a un nuevo contexto global menos favorable al libre comercio, uno de los pilares fundamentales del mecanismo. Además de la apertura conjunta de sedes diplomáticas, una de las innovaciones más celebradas de la AP, sobresalen el incremento sostenido en el número de países observadores, los avances en la formalización de actividades de cooperación con estos países, y más aún se destacan la creación de la categoría de "país asociado" en 2017, las negociaciones lanzadas con los cuatro primeros candidatos, Australia, Canadá, Nueva-Zelanda y Singapur, la postulación oficial de Corea del Sur y las negociaciones con Ecuador. En paralelo, la AP ha seguido avanzando en su acercamiento y búsqueda de convergencias con la ASEAN (Asociación de Naciones del Sureste de Asia, por su sigla en inglés), el MERCOSUR (Mercado Común del Sur) y la Unión Europea, y tres países de la AP -Chile, México y Perú se incorporaron al CP-TPP (Comprehensive and Progressive Agreement for Trans-Pacific Partnership).

En materia de cooperación, existen avances desiguales: por un lado, en materia de fomento empresarial, hay un gran dinamismo, y se han sostenido diversas iniciativas organizadas por las agencias nacionales de promoción comercial y de inversiones, como las Macrorruedas de Negocios, las Macrorruedas de Negocios del Turismo, los Foros de Oportunidades de Inversión, los Foros de Emprendimiento e Innovación (LAB4), los Foros de Empresarias Líderes, destinadas a estimular los contactos entre empresas y las inversiones intra-Alianza. La Plataforma de Intercambio Estudiantil y Académico, con metas modestas, constituye otra iniciativa exitosa, con XI convocatorias desde los inicios de la AP. Se le añadió más recientemente un programa de Voluntariado Juvenil. Por otro lado, es notable la incorporación de nuevos temas a las iniciativas de cooperación entre los miembros.

En contraste, la cooperación en el tema crucial de la infraestructura ha avanzado más lentamente. En primer lugar, cabe resaltar que los países han elevado su nivel de compromiso inicial, que era bastante bajo ${ }^{5}$, con la suscripción en mayo de 2013 (VII Cumbre) de un Acuerdo para el Establecimiento del Fondo de Cooperación de la AP. En segundo lugar, las instrucciones dadas

5 El texto del Memorando señalaba que las partes sólo iban a "procurar financiar las actividades de cooperación" y "hacer su mejor esfuerzo para sufragar los gastos" (Alianza del Pacífico 2011b: 3).
La Alianza del Pacífico a ocho años de su creación. Balance crítico y perspectivas Geneviève Marchini 


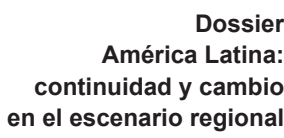

Dossier continuidad y cambio en el escenario regional en la VIII Cumbre por los mandatarios para "evaluar la creación de un Fondo para el desarrollo de infraestructura ... que incluya la posibilidad de captar aportes de fondos extranjeros" (Alianza del Pacífico 2014a: anexo 2) dieron paso a estudios de factibilidad de parte del BID y de la CAF, y a la declaración de creación del Fondo de Infraestructura de la Alianza del Pacífico (FIAP) en la XIII Cumbre (2018). El fondo se planteó como un vehículo gestionado por un gestor privado, con un capital de 1000 millones de US\$, a partir del cual se financiarán proyectos sobre la base de Asociaciones Público-Privadas. El bajo dinamismo de este aspecto de la cooperación, tan esencial para lograr una mayor interdependencia económica entre los países miembros, podría impactar de manera muy negativa los objetivos de mayor integración y en especial, de fortalecimiento de las cadenas de valor regionales.

\section{La Alianza del Pacífico: el debate acerca de sus objetivos y su caracterización}

La centralidad de las metas económicas para la Alianza llevó algunos analistas a afirmar que ésta retomaba los objetivos característicos del "regionalismo abierto" de los años 1990, concretizado en organismos como la CAN (1996, anteriormente Pacto Andino, creado en 1969) o el MERCOSUR (1991). Así Malamud (2014) plantea que la AP "reintrodujo el carácter central del comercio en la integración...” (Malamud 2014), y Briceño (2012) escribe que ésta constituye "el último bastión" del regionalismo abierto en América Latina (Briceño Ruiz 2012; citado en Leví, Reggiardo 2016: 191). Ciertamente, la AP se diferencia netamente del regionalismo post-hegemónico, post-liberal o alternativo (Rocha 2018a) que floreció desde mediados de los años 2000 en América del Sur, como producto de la pérdida de confianza en el modelo de regionalismo abierto y de la llegada al poder en varios países (Argentina, Brasil, Bolivia, Ecuador, Venezuela en particular) de gobiernos ubicados a la izquierda en el espectro político. Las instituciones que lo encarnaron, el ALBA (Alianza Bolivariana para los Pueblos de Nuestra Américas 2004), y la UNASUR (creada en 2004 como Comunidad Suramericana de Naciones, transformada en Unión de Naciones Suramericanas en 2008) privilegiaron temas poco tratados por el regionalismo abierto, como la paz, la infraestructura física, la energía, la seguridad, la arquitectura financiera, los aspectos sociales y políticos, abordados desde mecanismos de cooperación Sur-Sur (Leví, Reggiardo 2016; Rodríguez 2015) y con un enfoque de autonomía regional (Preciado 2018, Rocha 2018a). ${ }^{6}$

Sin embargo, ésta resulta ser una visión algo reductora pues la AP no se limita a la liberalización de flujos económicos, sino que incluye explícitamente iniciativas de cooperación que abarcan varios de los temas importantes del

6 Rocha (2018b) analiza el rol de la CELAC (Comunidad de Estados Latinoamericanos y Caribeños) en la construcción de esta región más autónoma. 
regionalismo post-hegemónico o alternativo: infraestructura física y conectividad, energía, financiamiento, intercambios educativos, aspectos sociales, seguridad. Ello lleva entonces a Rodríguez (2015) a afirmar que la Alianza conjunta elementos de las etapas anteriores del regionalismo latinoamericano: la liberalización comercial intrabloque del regionalismo abierto y la convergencia y concertación en temas políticos y sociales del regionalismo postliberal, creando sinergias entre ambos. A la vez innovaría "en cuanto considera tan importante como lo anterior aumentar y hacer más competitivo el comercio extrabloque, principalmente con el Asia-Pacífico" (Rodríguez 2014: 34-35), afirmación debatible, pues el regionalismo abierto privilegiaba también un crecimiento orientado hacia afuera. A la vez se puede plantear que al establecer como meta la creación o el fortalecimiento de cadenas productivas regionales, la AP retoma el rol atribuido a la integración regional por la CEPAL desde los tiempos de la industrialización sustitutiva de importaciones: "una ampliación del mercado y de la base de recursos productivos que permitiría aprovechar las economías de escala y las ventajas de la especialización y complementación industrial" (Briceño, Quintero, Ruiz de Benítez 2013: 11), pero en un contexto radicalmente distinto de apertura e integración en Cadenas Globales de Valor (CGVs).

Ciertamente, es clara también la influencia de las estructuras de la región Asia-Pacífico y en particular de APEC -Asia-Pacific Economic Cooperation, por su sigla en inglés- (Leví, Reggiardo 2016: 204; Rodríguez 2014: 34-35). Aunque las instituciones de la AP son más formales que las de este organismo, pues requieren acuerdos de liberalización comercial previos entre los miembros, divergen claramente del regionalismo abierto latinoamericano de los años 1990 en su institucionalidad liviana y en el rechazo a toda cesión de soberanía (Garzón, Nolte 2018: 343). A la vez, se acercan a los procesos vigentes en APEC en su manera de abordar en forma flexible los temas que se incluyen en el proceso de integración. ${ }^{7}$ Estas características han sido apreciadas de manera muy positiva por analistas anglo-sajones o europeos cercanos a los mercados financieros o a instituciones internacionales, quienes consideraron que la Alianza constituía un mecanismo pragmático, flexible, favorable a las empresas y antecedido por flujos de inversiones e intereses privados (McKeigue 2012; Donnan 2015; Echebarría, Estevadeordal 2014: 33). La ausencia de "grandes objetivos" (Torres 2016, Rathborne 2014), el enfoque de tipo early harvest o "paso por paso" de los acuerdos tomados por la AP contribuyen al éxito del mecanismo (Dade, Meacham 2013: 5; Regueiro 2014) ${ }^{8}$. La AP constituiría el "lado práctico" de América Latina (Keppel 2015).

Ahora bien, más allá de estos aspectos generales, la AP presenta caracte-

\footnotetext{
7 Este tema se retoma y discute más adelante.

8 Así, "The Economist" opinaba: "Behind the excitement is the sense that the PA is a hard-nosed business deal, rather than the usual gassy rhetoric of Latin American summitry" (The Economist 2013). Dade y Meacham (2013) consideraban que la AP aprendió las lecciones de los fracasos pasados de la integración latinoamericana (Dade, Meacham 2013: 5).
}

La Alianza del Pacífico a ocho años de su creación. Balance crítico y perspectivas Geneviève Marchini 


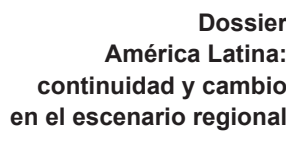

en el escenario regiona rísticas específicas que la distinguen de las categorías convencionales de mecanismos de integración regional y que han generado intensos debates en la academia regional acerca de sus propósitos y de su alcance.

\section{Visión económica compartida y ausencia de continuidad territorial}

Una primera particularidad de la AP frente al regionalismo latinoamericano anterior a ella es la ausencia de continuidad geográfica entre los países fundadores. Con el ALBA, la AP es el primer organismo subregional cuyos miembros no pertenecen a la misma subregión (Garzón, Nolte 2018: 345). Esta característica es en parte involuntaria, producto de las divergencias ideológicas que contribuyeron al fracaso de la iniciativa anterior del Arco del Pacífico, que reunía todos los países de la región ribereños del Pacífico latinoamericano. A la vez, marca el regreso de México como actor en América del Sur (George 2014: 28, Malamud 2014, Nolte 2016: 1). ${ }^{9}$ Más que la cercanía geográfica, es por lo tanto la visión compartida en materia de políticas económicas y de inserción internacional que constituye el cimiento de la $\mathrm{AP}^{10}$ De hecho, como lo hemos visto, si la declaración de Lima menciona la región latinoamericana como ámbito de reclutamiento de nuevos miembros, el art. 11 del Acuerdo Marco parece sugerir que la membresía de la AP está abierta a países extrarregionales que compartan esta visión.

En la base de la rápida sucesión de acuerdos tomados por la AP, están los antecedentes de políticas económicas similares. Se trata, en primer lugar, de políticas monetarias y fiscales ortodoxas. Aun cuando se pueden discutir los costos de estas políticas en términos de crecimiento, desarrollo y equidad social, es innegable que permitieron alcanzar un grado apreciable de estabilidad macroeconómica -inflación baja, déficits fiscales controlados, reducción o control del endeudamiento público, ausencia de grandes desequilibrios externos- aún en tiempos de turbulencias externas. En segundo lugar, los cuatro países comparten una estrategia de crecimiento "hacia afuera" y de apertura comercial y financiera dominada desde 2000 por la firma de numerosos acuerdos de liberalización comercial bilaterales con socios ubicados cada vez más fuera del continente americano y, en particular, en Asia-Pacífico. En suma, la AP reúne los países de América Latina que más han apostado por la vía recomendada por el Consenso de Washington "aumentado". Así, la Alianza constituiría una "colaboración natural entre países que piensan igual y que han aplicado de manera

9 Con el surgimiento de la AP, la integración regional vuelve a concebirse como un proyecto de alcance latinoamericano, y no solamente sudamericano, resolviéndose de esta manera la cuestión de "qué" integrar (Malamud 2014). A la vez, el regreso de México como actor regional constituye un desafío a una hegemonía regional de Brasil.

10 Rathbone, editor para América Latina del "Financial Times" afirmaba "The PA is more of an attitude than a physically coherent group" (Rathbone 2015). Entre otros aspectos, destacaba una actitud común respecto al rol del Estado en la economía y frente a la inserción en la economía internacional. 
independiente reformas y estrategias similares, con un ojo hacia el Este tanto como socio comercial como modelo para el desarrollo" (George 2014: 27).

Los requisitos previos de carácter político - respeto a la democracia y a los derechos humanos, separación de poderes, etc.- puestos a la incorporación de nuevos miembros anclan la AP en el campo occidental. Ramírez y León (2014) afirman que la ausencia de la palabra "económico" en el Acuerdo Marco permitiría calificar la AP como un acuerdo "de carácter político amplio" (Ramírez y León 2014: 36). Así, a diferencia de Dade y Meacham (2013), para quienes no cabe buscar ideología alguna en la Alianza, ésta no es neutral ideológicamente, sino liberal o neoliberal (Ardila 2015: 245; Saltalamacchia, Urzúa 2016: 115). Si bien la Alianza "no es una estructura de carácter político en si misma [...] sí refleja los intereses y la decisión política al más alto nivel [...] sobre todo en aspectos como la perspectiva de desarrollo e inserción global neoliberal" (Leví, Reggiardo 2016: 197). En el mismo tenor, Ramírez (2016) plantea que aquello que generó tanto interés hacia la Alianza en Europa y las Américas, no es tanto lo económico como los principios políticos e ideológicos que sustentan la membresía de la AP (Ramírez 2016: 155). Rocha y Morales (2015) precisan que la Alianza tiene dos ejes geoeconómicos y geopolíticos: el de América del Norte predomina, materializado en la presencia de México, en la participación de este país en el TLCAN (Tratado de Libre-Comercio de América del Norte), y en los Tratados de Libre-Comercio (TLCs) firmados por los demás miembros con EE.UU. "El eje del Pacífico asiático es todavía secundario y complementario, si bien constituye la apuesta principal." (Rocha, Morales 2015: 141-142).

En materia de política exterior, la visión compartida por los miembros de la Alianza se traduce, en particular, en la libertad acordada a éstos para seguir negociando acuerdos comerciales en forma independiente y en la decisión de respetar todos los acuerdos vigentes, incluso entre ellos mismos -cabe recordar que Colombia y Perú siguen siendo miembros de la CAN. Esta característica se contrapone al regionalismo abierto de la propia CAN y del MERCOSUR, que se abocaron a construir un arancel externo común y establecieron el compromiso de negociaciones en bloque, con los riesgos que este mayor nivel de exigencia supone. ${ }^{11}$ En este sentido, vale subrayar que el art. 8 del Acuerdo Marco no contribuye a simplificar el spaghetti bowl o noodle bowl de acuerdos comerciales de la Alianza, pues el Acuerdo Marco se superpone a los tratados previos mas no los reemplaza.

\section{El acercamiento a Asia-Pacífico como objetivo central}

La segunda especificidad de la AP es el hecho de definirse en parte por la relación que busca desarrollar con otra región del mundo, Asia-Pacífico (Bárcena

${ }^{11}$ Esta cláusula llevó la CAN a la crisis y el MERCOSUR a una paralización de sus negociaciones externas.
La Alianza del Pacífico a ocho años de su creación. Balance crítico y perspectivas Geneviève Marchini 
Dossier América Latina: continuidad y cambio en el escenario regional
2016, Rodríguez 2015). ${ }^{12} \mathrm{Al}$ igual que en el punto anterior, este objetivo original se fundamenta en -y busca en la práctica consolidar- políticas anteriores a la creación de la Alianza. En efecto, tres miembros de la AP pertenecen al foro APEC. Chile es miembro desde 1994, México desde 1993 y Perú desde 1998, mientras Colombia, que no pudo ingresar debido a la moratoria establecida por el organismo, pertenece, al igual que los demás miembros de la Alianza, a otros mecanismos transpacíficos como el foro empresarial PBEC (Pacific Basin Economic Council, desde 1994) o el organismo tripartita gobierno-academia-empresas PECC (Pacific Economic Cooperation Council, desde 1995). ${ }^{13}$ Los tres países miembros de APEC participaron en las negociaciones del TPP (Trans-Pacific Partnership) que incluía, además de Canadá y Estados Unidos, siete países de Asia-Pacífico: Australia, Brunéi, Malasia, Japón, Nueva Zelanda, Singapur y Vietnam, lo que les llevó a integrarse a su sucesor, el CP-TPP, ya sin Estados Unidos.

Por otro lado, después de la firma del TLCAN entre México, Estados Unidos y Canadá, entrado en vigencia en 1994, los países miembros de la Alianza del Pacífico han encabezado la firma de TLCs o de Acuerdos de Asociación económica (AAEs) con naciones de Asia a partir de 2000. Ravenhill (2011) ubica el florecimiento de estas agrupaciones "transregionales" en un contexto global marcado por el fin de la Guerra Fría, por una mayor interdependencia global y por el ascenso de la ideología neoliberal (Ravenhill 2011: 189). Garzón (2015) las sitúa en el contexto de un mundo multipolar, y plantea que este "regionalismo cruzado" (cross regionalism) ${ }^{14}$ puede traer distintas ventajas a los países firmantes. Además de un mejor acceso a los mercados globales o una mayor atracción de Inversión Extranjera Directa (IED), permitiría "reducir la dependencia económica de un solo socio comercial grande" y hacer más visibles a los países pequeños o medianos, pues éstos se pueden transformar "en hubs de TLCs que cubren varias regiones" (Garzón 2015: 11).

Después del retiro de EE.UU. del TPP y ante el nuevo entorno global, Pastrana y Castro afirman que la AP se ha posicionado como "uno de los ejes articuladores de un interregionalismo pos-TPP" (Pastrana, Castro 2017: 76). Este planteamiento se deriva, en particular, de la mencionada creación de parte de la AP de la categoría de Estado Asociado, anunciada en la Cumbre de Cali, en junio de 2017, y del lanzamiento inmediato de negociaciones conjuntas con Australia, Canadá, Nueva Zelanda y Singapur, los cuatro primeros países en postular a esta categoría. Asi-

12 Bárcena notaba correctamente que la AP "no estaba concebida por sus miembros como un foro para negociar conjuntamente acuerdos comerciales o de inversión con otros países o agrupaciones" (Bárcena 2016: 60). En 2017, el nuevo contexto global llevó la Alianza a introducir un cambio fundamental que autorizó estas negociaciones conjuntas.

13 Los cuatro miembros de la AP participan también, con un nutrido grupo de países de América Latina, al Foro de Cooperación de América Latina y Asia del Este (FOCALAE) creado en 1999. Este foro reúne 20 países latinoamericanos y 16 países de Asia del Este.

14 El autor lo define como "una política llevada a cabo de manera individual por Estados que establecen áreas de libre comercio a pequeña escala con Estados ultramarinos” (Garzón 2015: 9). 
mismo, para los autores, la participación de Chile, México y Perú en el CP-TPP permitiría "conectar comercial y económicamente, de manera más profunda, los Estados del continente americano y del Asia-Pacífico” (Pastrana, Castro 2017: 76).
La Alianza del Pacífico a ocho años de su creación. Balance crítico y perspectivas

Geneviève Marchini

\section{La "integración profunda": un concepto equívoco}

En tercer lugar, la AP difiere del regionalismo latinoamericano existente tanto en lo que se propone - profundidad, amplitud-como en la manera en que busca alcanzar estos objetivos, y en las instituciones que deben guiar y apoyar el proceso de integración. Sus características hacen del mecanismo algo difícilmente clasificable, y para el cual se han buscado aplicar nuevas categorías de análisis. Como lo hemos visto, la AP plantea crear un "área de integración profunda” con miras a avanzar hacia la libre circulación de bienes, servicios, capitales y personas. El debate se centra en ¿qué se entiende por "área de integración profunda" ?, ¿Hacia dónde y cómo busca avanzar la AP?, ¿Es posible alcanzar estos objetivos con la institucionalidad ligera que adoptó el mecanismo?

Varios autores han buscado definir la naturaleza de la Alianza al relacionarla con las etapas "clásicas" de la integración, definidas por Bela Balassa (Balassa 1964, en: Vieira 2015). Estas etapas se utilizan para clasificar los mecanismos de integración regional y van desde el primer nivel de integración, la zona de libre comercio basada en la liberalización comercial, hasta el nivel más avanzado, el de unión económica con armonización de políticas, pasando por las etapas de creación de un arancel externo común y de una unión aduanera, la que constituye la primera cesión de soberanía, seguida del mercado común con libre circulación de los factores de producción. (Tremolada 2016: 76-77, Vieira 2015: 72-73).

La integración profunda que busca promover la Alianza no coincide plenamente con las etapas clásicas de la integración, aun cuando la propia AP se refiere a éstas cuando proclama haber lanzado una "zona de libre comercio" después de la entrada en vigencia del Protocolo Adicional, y cuando el objetivo de libre circulación parece referirse a un mercado común con libre circulación de factores, las "cuatro libertades" (Tremolada 2016).${ }^{15}$ En efecto, el concepto de "integración profunda" tiene un contenido equívoco (Vieira 2015).

Inicialmente, se concibió como una definición fuerte de integración. Schiff y Winters (2003) aplican el término a "acuerdos [...] que buscan realizar algún nivel de unión económica y que crean instituciones supranacionales para alcanzar este objetivo" (Schiff, Winters 2003: 150). ${ }^{16}$ Consideran que la inte-

\footnotetext{
15 Este concepto nace con la creación del Mercado Único Europeo.

16 El concepto se refirió inicialmente a la construcción de la integración europea, y específicamente a la creación del Mercado Único. El concepto habría sido introducido por primera vez en Lawrence y Littan (1990) (Schiff, Winters 2003: 2.150).
} 
Dossier América Latina: continuidad y cambio en el escenario regional gración profunda es afín al concepto de “integración positiva”, acuñado por Tinbergen (1954), e incluye "esfuerzos positivos para coordinar políticas". En este tenor, Rocha y Morales (2015) definen la integración profunda como "la conformación de un sistema de integración multidimensional político, económico, social, cultural y espacial” (Rocha, Morales 2015: 109). Definida de esta manera, la "integración profunda" contrasta con el nivel de "integración superficial” (shallow integration), consistente en la eliminación de obstáculos y en el otorgamiento del trato nacional en materia comercial, el que es cercano a la "integración negativa" del mismo Tinbergen (1954). ${ }^{17}$

Ahora bien, el término "integración profunda" ha pasado más recientemente a aplicarse de forma bastante más laxa a los acuerdos comerciales preferenciales (ACP) que se multiplicaron desde mediados de los años 1990, en un contexto global marcado por el estancamiento de las negociaciones multilaterales de comercio, y su sentido se ha vuelto más impreciso y menos fuerte. Los ACPs más recientes, entre los cuales se cuenta la Alianza del Pacífico, buscan una "integración profunda" que incluye, además de la eliminación de barreras "a la frontera" (shallow integration), una serie de "disciplinas" más "profundas", que buscan regular las actividades, hasta en el seno de la economía doméstica. ${ }^{18}$ Estas disciplinas introducen requisitos más exigentes que los acordados en la OMC (Organización Mundial del Comercio) -en este caso se conocen bajo los términos de "OMC-Plus"-, o que no están contemplados por los acuerdos multilaterales -calificados como "OMC-X". Entre éstos, destacan temas como la protección de la propiedad intelectual, la protección y el fomento de la inversión extranjera, el acceso a las compras gubernamentales, la armonización de regulaciones o las medidas para combatir la competencia desleal, todos estos temas esenciales para la organización internacional de la producción, en especial manufacturera, en CGVs. ${ }^{19}$ Regueiro (2014) subraya que estos temas pertenecían al "núcleo duro" de la fracasada ALCA (Área de Libre Comercio de las Américas), el que creó mayor nivel de resistencias entre los participantes latinoamericanos: inversiones, servicios, propiedad intelectual y compras gubernamentales (Regueiro 2014: 164).

En suma, las disciplinas integradas en los ACPs influyen cada vez más en las políticas internas de sus miembros y la "integración profunda” pasó a definirse como la aceptación de que “... reglas internacionales gobiernen

${ }_{17}$ Barbosa y Buitrago (en prensa) retoman la definición de Schiff y Winters (2003), aplicando criterios empíricos para determinar el grado de profundidad de la integración que se plantea la AP.

${ }_{18}$ Lawrence (1996) constituye un antecedente para el análisis de este fenómeno. (Lawrence, 1996: 17, en Young 2017: 454).

19 La fragmentación de la producción, a través de la cual las grandes firmas manufactureras externalizan tareas y deslocalizan las etapas de la elaboración de un producto con el fin de reducir costos, requiere y produce numerosos intercambios internacionales de dos vías entre las economías participantes: "El comercio del siglo XXI es un conjunto complejo e interconectado de flujos transfronterizos de bienes, inversiones, tecnología, servicios, personal técnico y de dirección, y capital” (Baldwin 2013: 39). 
las políticas domésticas con el fin de mitigar sus efectos adversos sobre el comercio (Young 2017: 452, 455). En la terminología de Schiff y Winters (2003), no se trata de integración profunda sino de un nivel menos exigente de "integración de políticas" (policy integration), que implica, en el mejor de los casos, "un nivel intermedio de integración que incluye una cooperación gubernamental cercana para armonizar las reglamentaciones internas y las políticas, pero que no contempla una autoridad supranacional" (Schiff, Winters 2003: 20). Así el uso de parte de la AP del término "integración profunda" ubica el acuerdo en el ámbito de los ACPs de nuevo cuño y señala su afinidad con las recomendaciones de organismos multilaterales referidas a la inserción en CGVs de los países latinoamericanos. A la vez, deja bastante indefinido el grado de profundidad al cual se quiere llegar, el que sólo se podrá determinar analizando las políticas acordadas e implementadas en cada mercado, tanto en la frontera, como más allá de la frontera -eliminación de leyes y reglamentos que inhiben los intercambios internacionales de bienes y servicios-, y de forma transfronteriza -armonización de medidas regulatorias- (Lloyd 2008). ${ }^{20}$ La mayoría de estas medidas se ubica en las categorías de "integración superficial" o eliminación de obstáculos y trato nacional, pero algunos procesos en curso en la AP -la integración financiera, el FIAP, entre otros- muestran un potencial para ir más allá.

\section{La caracterización de la Alianza del Pacífico como mecanismo de integración: aportaciones del debate académico}

Lo anterior permite entender que la literatura que analiza la AP muestra apreciaciones contrastadas acerca de la profundidad relativa del área de integración que se busca constituir. Muchos autores clasifican la Alianza como Acuerdo o Zona de Libre Comercio caracterizada por un tipo de integración negativa o superficial o regionalismo económico superficial (Tremolada 2016; Garzón, Nolte 2018). Rocha y Morales la consideran como un TLC de banda ancha, que sigue el modelo del TLCAN (Rocha, Morales 2015) y Regueiro (2014) como un TLC-plus. Vieira (2015) clasifica la AP como un área de libre comercio de segunda generación, que además de rebajas arancelarias, incluye la eliminación de barreras no-arancelarias, la armonización de normas, la eliminación de obstáculos y, además de lo anterior, provisiones sobre inversión y propiedad intelectual. Añade que, al desaparecer la etapa de unión aduanera con arancel externo común, debido a la disminución o desaparición internacional de las tarifas, "la fase de mercado común referente a la circulación de todos los factores de producción [...] se ve muchas veces subsumida en los compromisos de los tratados de libre comercio,

${ }^{20}$ Lloyd propone una metodología basada en estas categorías y analiza los grados de integración y los "estilos" de distintos acuerdos comerciales (Lloyd 2008).
La Alianza del Pacífico a ocho años de su creación. Balance crítico y perspectivas Geneviève Marchini 
Dossier América Latina: continuidad y cambio en el escenario regional los cuales mezclan la apertura comercial con la circulación de los factores". Pero ello de ninguna manera implicaría integración profunda porque ésta se refiere a un mercado común o una unión económica con armonización de políticas (Vieira 2015: 72-73).

Saltalamacchia y Urzúa (2016) plantean que "la AP no es, desde el punto de vista de la economía política, un ejercicio de integración profunda pero tampoco es un simple acuerdo comercial. Se trata quizás de un nuevo animal político en el panorama del regionalismo, algo parecido a un área de libre comercio plus, aderezada con iniciativas educativas, culturales y diplomáticas" cuyo núcleo consistiría en acuerdos de liberalización y acuerdos de cooperación (Saltalamacchia, Urzúa 2016: 105).

Barbosa y Buitrago (en prensa) y Rodríguez (2015) califican respectivamente la AP de "regionalismo estratégico" y de "regionalismo estratégico hacia afuera”, y destacan una dimensión adicional: desarrollar una ventaja competitiva para los países miembros, fundamentada en el peso geopolítico de la Alianza y en procesos de concertación y cooperación entre sus miembros (Barbosa, Buitrago en prensa; Rodríguez 2015). Para Rodríguez (2015) “aumentar el comercio intrabloque ya no es el objetivo principal, pues se comprende que la libre circulación de los factores productivos es condición necesaria para aumentar y hacer más competitivo el comercio extrabloque, principalmente con el AsiaPacífico" y este proceso autorizaría “internacionalizar sus economías de forma competitiva e integrada a la economía global, en lo que se denomina regionalismo estratégico 'hacia afuera' " (Rodríguez 2015: 43-45).

La dimensión estratégica es resaltada también por Garzón y Nolte (2018), quienes afirman que la AP no constituiría "un retorno a las prácticas de gobernanza del regionalismo abierto (Briceño-Ruiz 2014), sino un ejemplo paradigmático de la gobernanza minilateral en la región" (Garzón, Nolte 2018: 334). Para los autores, el "minilateralismo" "reúne un grupo pequeño de países que piensan igual, independientemente de la región geográfica a la cual pertenecen ...Lo que cimienta estas coaliciones no es la geografía sino objetivos comunes y un interés en un área específica de la gobernanza" (Garzón, Nolte 2018: 333). ${ }^{21}$

La AP tendría un interés exclusivo en la gobernanza económica y sus miembros practicarían "una forma de 'marca-país' conjunta para promover el comercio y la inversión, y elevar su estatus internacional y su visibilidad” (Nolte 2016: 1). El principal objetivo estratégico de este instrumento minilateral no sería tanto el fortalecimiento del comercio entre los socios como la atracción de una mayor proporción de la Inversión Extranjera Directa (IED) orientada a América

${ }^{21}$ El minilateralismo muestra tres características principales: 1. Reúne el número más pequeño de países necesario para alcanzar una meta particular (Naím 2009); 2. A menudo se trata de acuerdos más flexibles e informales (Brummer 2014); 3. Abordan los retos de manera desagregada y punto por punto (piece meal) y no con un acuerdo de forma global (Garzón, Nolte 2018: 333). 
Latina, a través de una diferenciación de sus miembros (Garzón, Nolte 2018: 345). La diferenciación y la marca-país de la Alianza, fundamentadas en las políticas de sus miembros hacia el libre mercado y el Estado de derecho y en su récords de crecimiento económico, demostraría que poderes medios (México) y poderes regionales secundarios (Colombia y Chile) pueden cooperar y crear organismos regionales con el objetivo de elevar su estatus internacional (Garzón, Nolte 2018: 345). El énfasis en el tamaño agregado de la economía de la AP, o de sus exportaciones y su posicionamiento a nivel global, forma parte de esta estrategia, reflejada en la comunicación de la Alianza.

El éxito de la "marca" Alianza del Pacífico ha encontrado ecos en numerosas publicaciones. George, quien acuñó el término los "Pumas del Pacífico", afirmaba "a través de la Alianza del Pacífico, los Pumas han agregado peso a su impulso individual y han establecido su modelo como un enfoque atractivo para muchos países latinoamericanos pequeños y medianos" (George 2014: 27). La marca AP ha suscitado expectativas a menudo exageradas en el ámbito financiero internacional ${ }^{22}$, pero no exentas de dudas. Así, la revista "The Economist" la calificaba en 2013 de "una muestra brillante de marketing diplomático" (The Economist 2013), pero la misma revista en la columna Bello, publicaba en 2015 que la AP era "una gran marca en busca de un producto común” (Bello 2015). McKeigue, en "MoneyWeek", se hacía eco del escepticismo de los mercados, en razón de la proliferación de acuerdos regionales en América Latina (McKeigue 2012, McKeigue 2014), pero también debido a las propias características de sus miembros: países como Colombia y Perú les parecen a menudo pequeños y arriesgados a los inversionistas (McKeigue 2013), y México es a perennial underachiever, con décadas de crecimiento mediocre (McKeigue 2012). Saltalamacchia y Urzúa (2016) concluían: "La exageración de las expectativas en torno a la AP es el fruto de uno de sus logros incontrovertibles: colocar a sus países en una vitrina atractiva y con ello entrar en el imaginario del mundo financiero" (Saltalamacchia, Urzúa 2016: 118). En la sección siguiente, examinaremos las expectativas que se generaron acerca del potencial de la Alianza del Pacífico y las condiciones que se consideran necesarias para cumplir con ellas.

\section{Expectativas y condiciones necesarias para el éxito}

¿Qué se espera de la Alianza del Pacífico en la literatura que la analiza? En este apartado, abordaremos, en primer lugar, las expectativas y los retos relacionados con los objetivos explícitos de integración regional interna de la AP

22 McKeigue, en la revista "MoneyWeek" presentaba a la Alianza como un "nuevo superpoder latinoamericano" (McKeigue 2013) integrado por las "economías más prometedoras de América Latina" (McKeigue 2012). Moisés Naím los calificaba de "most successfull economies in LA" (Naím 2014).
La Alianza del Pacífico a ocho años de su creación. Balance crítico y perspectivas Geneviève Marchini 
Dossier

América Latina:

continuidad y cambio

en el escenario regional y en un segundo momento analizaremos su proyección hacia el Asia-Pacífico y la propia región latinoamericana.

\section{Integración intra-Alianza, retos económicos y relaciones económicas con América Latina y Asia}

Lo que se espera de la Alianza se relaciona, sobre todo, con los aspectos económicos que constituyen su propósito principal. Comercio e inversiones intraAlianza, cadenas de valor regionales y vinculación con CGVs, diversificación de las exportaciones y de la estructura productiva, elevación de la productividad constituyen los temas más abordados. La literatura señala que las oportunidades existen, sin embargo, los obstáculos son considerables.

En efecto, si bien las economías de la AP han mostrado cierto éxito, éste es relativo, en particular si se compara con la experiencia de los países de Asia del Este. Ghymers argumenta que la convergencia en ingresos de las economías de América Latina con las economías desarrolladas, observada desde la década de 2000, no es sustentable, pues no se fundamentó en una convergencia comparable de los elementos impulsores del crecimiento ${ }^{23}$-Colombia constituye la excepción más notable, seguida de Chile. Más aún, las economías latinoamericanas como conjunto mostraron divergencia en indicadores de desarrollo como seguridad personal, calidad del medioambiente o igualdad de oportunidades por género entre 2004 y 2013 y en este sentido los miembros de la AP no se distinguen de las demás economías de la región. Se habría producido un crecimiento sin desarrollo (Talvi 2014, en: Ghymers 2016). ${ }^{24}$ El buen desempeño en materia de crecimiento fue el producto de condiciones externas muy favorables y no repetibles -alta demanda de China, políticas monetarias acomodaticias de los países desarrollados.

En la base de las pobres perspectivas de crecimiento y desarrollo en la región se encuentra el estancamiento de la Productividad Total de los Factores (PTF), que en 2011 no superaba los niveles de 1990 (Ghymers 2016: 208). Además de condiciones estructurales internas, esta tendencia se explica por una débil inserción internacional de las economías latinoamericanas, entre éstas las de los miembros de la AP (Ghymers 2016, Marchini 2015). Si bien han expandido sus exportaciones y sus economías son ahora más abiertas, con

${ }^{23}$ El método de análisis de la convergencia económica de la Brookings Institution (Talvi 2014), que Ghymers utiliza, contempla cinco factores impulsores del crecimiento: integración comercial, infraestructura física y tecnológica, capital humano, innovación y calidad de los servicios públicos.

${ }^{24}$ Los indicadores de convergencia del desarrollo incluyen el ingreso relativo, que aumenta para el conjunto de países considerados y el indicador agregado de desarrollo, que disminuye debido al deterioro señalado en los tres indicadores mencionados en el texto, siendo el de la igualdad de oportunidades por ingreso el único componente al alza. El índice se calcula con datos de siete economías latinoamericanas: Argentina, Brasil, Chile, Colombia, México, Perú y Venezuela (Ghymers 2016: 208). 
un mayor peso del comercio exterior (exportaciones + importaciones) en relación con el Producto Interno Bruto, los tres países andinos han profundizado su carácter primario-exportador y México, el único de los socios en haber modificado su inserción internacional, posee una plataforma exportadora de manufacturas poco vinculada con el resto de su economía, que agrega poco valor nacional y genera poco crecimiento (Marchini 2015). Ramírez y León (2014) plantean que las políticas económicas liberales practicadas por estos países durante los últimos treinta años han dañado su potencial económico al impactar negativamente su sector manufacturero y avanzan que "... las cuatro economías de la AP han retrocedido y ahora pueden ser comparadas con las economías menos desarrolladas del sureste de Asia”. (Ramírez, León 2014: 3).

En este contexto, ¿qué puede aportar la Alianza del Pacífico? En materia de comercio intra-Alianza, existen puntos de vista contrapuestos: algunos analistas consideran que, partiendo de un nivel bajo -la importancia relativa de los socios prácticamente no supera el 10 u $8 \%$ del comercio exterior, y se establece en menos de $2 \%$ para México-, existe un gran potencial para desarrollarlo (Naím 2014). El comercio intrarregional presenta además múltiples bondades: los intercambios incluyen más bienes de mayor valor agregado y contenido tecnológico que contribuyen a diversificar las exportaciones más allá de las materias primas -en especial para los países andinos- y es más fácil que las MPyMEs se incorporen. Sin embargo, otros autores plantean que existe poco margen para aumentar los intercambios de bienes, pues las especializaciones relativas de las economías no las hacen complementarias, las distancias son grandes (Nolte 2016: 5) y la infraestructura deficiente. A la vez, una proporción elevada del comercio intrarregional ya estaba liberalizada en los TLCs bilaterales previos a la creación de la AP. Por estas razones, varias contribuciones avanzan que el mayor impacto de la AP podría provenir más bien de la liberalización de los flujos de capitales (George 2014: 28, Nolte 2016: 5), y de la mayor atractividad para la Inversión Extranjera Directa, en particular desde Asia (Bello 2015; Garzón, Nolte 2018: 345; Saltalamacchia, Urzúa 2016).

La esperada mejora de la productividad en los países miembros es un tema crucial: es "el Santo Grial que ha escapado por mucho tiempo a la mayoría de las economías latinoamericanas" (McKeigue 2014). Se espera que las reformas introducidas por el mecanismo puedan bajar los costos al dar a las empresas acceso a un mercado más grande, y a un conjunto más amplio de proveedores y de talentos (McKeigue 2012). Ahora bien, alcanzar este objetivo requerirá importantes inversiones, en infraestructura, física y digital, en energía, en equipos y tecnología, en educación de la población (McKeigue 2013, Heine et al. 2016, Kotshwar 2013). Cabría liberalizar pronto los $8 \%$ de partidas no incluidas en la desgravación inmediata para dar una señal positiva a los inversionistas (Naim 2014, Torres 2016). Ghymers (2015) plantea que se requerirá una estrategia específica para abordar el tema de la baja productividad y converger con los niveles de productividad de las economías desarrolladas (Ghymers 2016: 210).
La Alianza del Pacífico a ocho años de su creación. Balance crítico y perspectivas Geneviève Marchini 
Dossier América Latina: continuidad y cambio en el escenario regional
A su vez, el tema de la productividad se relaciona estrechamente con la reestructuración productiva y la creación y/o el fortalecimiento de las cadenas regionales de valor: éste es el real big deal de la Alianza del Pacífico (Keppel 2015). Como vimos, este tema que no figuraba explícitamente en los objetivos iniciales de la Alianza se incorporó en 2014 con la firma del Protocolo Adicional, y constituye un giro importante en la definición de integración regional que el mecanismo plantea. En consecuencia, ha dado lugar a numerosas contribuciones. Sobresale porque es un reto de gran magnitud: las publicaciones de organismos multilaterales -OMC, CEPAL, entre otros- relativas a CGVs subrayan generalmente la baja inserción de las economías de América Latina en las redes manufactureras -con la excepción de México y América Central- y la perspectiva negativa que ello plantea para sus expectativas de crecimiento económico y de diversificación exportadora. ${ }^{25}$ De empezar a cumplirse, la creación o el fortalecimiento de las cadenas productivas regionales transformaría profundamente la inserción internacional de los miembros del mecanismo.

Entre los beneficios más importantes que se pueden esperar sobresalen el de transformar la estructura productiva y la estructura de exportaciones de los países miembros para que incorporen una mayor proporción de productos manufacturados, y la creación de ganancias de competitividad a nivel internacional. Éstas se alcanzarían a través de "economías a escala transfronterizas y vínculos industriales para ayudar sus miembros a beneficiarse plenamente de la Cuenca del Pacífico [...] y seguir creciendo después del fin de auge de las materias primas" (Rathbone 2014). Marczak, George et al. (2016) concuerdan en que el desarrollo de cadenas regionales permitiría ir más allá de la dependencia de las materias primas y hacia "una integración en las intricadas cadenas de suministro que generan rápido crecimiento y desarrollo en la cuenca asiática del Pacífico" (Marczak, George et al. 2016: 13). Asimismo, Saltalamacchia y Urzúa (2016) afirman que el alza futura del comercio interregional dependerá en buena medida de la capacidad de los miembros de la Alianza en integrarse a las cadenas productivas de valor asiáticas en sectores específicos (Perry 2014: 10; en: Saltalamacchia, Urzúa 2016: 110-111).

En suma, superar el patrón primario-exportador de los países andinos -e incluso de México en sus intercambios con Asia del Este- constituye probablemente uno de los retos mayores de la AP, y, de producirse, podría ser una de las medidas concretas de su éxito (Bárcena 2016: 64). Ello implica que la Alianza deberá "convertirse en el marco para el aprendizaje y mejoramiento del establecimiento de CGV, aprovechando la acumulación de origen establecida para insumos de los países miembros" y llegar a la "formación de multilatinas con cadenas de valor intrarregional” (Vieira 2015: 86-90). Esta nueva dinámica autorizaría la generación de empleos de mayor calidad, el fortaleci-

25 Las economías de América del Sur, entre ellas Chile, Colombia y Perú, están insertas en CGVs, pero al inicio de estas redes productivas, como proveedores de materias primas. 
miento de la baja interdependencia regional, a la vez que facilitaría la inserción de las MPyMEs en actividades exportadoras.

Algunas contribuciones, que utilizan distintas metodologías, han identificado actividades donde existen vínculos intraindustriales y conexiones productivas entre los miembros de la AP. Hernández et al. (2015) encuentra que cada país miembro posee "entre 4 y 8 sectores de bienes intermedios y de capital (y otros tantos para los bienes finales) con potencial para aprovechar el desarrollo de la AP, a través del establecimiento de cadenas de valor, de la profundización de las exportaciones o a través de procesos de fusión y adquisición transfronterizos dentro de la AP". Entre estos sectores destacan los de plásticos y sus manufacturas, de papel y cartón y sus manufacturas, de máquinas y material mecánico; en los bienes de consumo, las actividades de perfumería y cosmética; los cereales, pasta, pastelería y harinas; y las preparaciones alimenticias (Hernández et al. 2015: 1-2). Durán y Cracau (2016) estiman que existen "espacios para la creación de cadenas de valor regionales en sectores como los plásticos, los alimentos, el papel y el cartón, los productos químicos y farmacéuticos, entre otros". (Durán, Cracau 2016: 47).

Por otro lado, Roldán, Castro y Eusse (2013) han analizado la presencia de comercio intraindustrial entre Asia del Este y la Alianza del Pacífico entre 2007 y 2011, a través del cálculo del Îndice de Grübel-Lloyd (IGL) ${ }^{26}$, y encuentran un nivel incipiente de comercio intraindustrial, que se concentra en las industrias de las TICs (Tecnologías de la Información y la Comunicación), en el sector automotriz, el cuidado de la salud, los textiles y confecciones y el sector electrónico (Roldán, Castro, Eusse 2013). Marchini (en prensa) analiza tanto el comercio intraindustrial intra-Alianza como los vínculos intraindustriales entre los miembros de la Alianza y sus tres primeros socios en Asia: China, Corea del Sur y Japón, estimados mediante el IGL calculado entre 2008 y 2014. ${ }^{27}$ Encuentra que existen conexiones intraindustriales entre todos los pares de países: en la propia AP se concentran entre los tres países andinos y entre México y Colombia. La actualización a 2017 (tabla 1) permite constatar que las conexiones intraindustriales se han mantenido e incluso fortalecido para los pares Chile-Perú o Colombia-México entre 2008 y 2017.

${ }^{26}$ El índice de Grübel-Lloyd o IGL (Grübel \& Lloyd 1975) busca estimar las conexiones intraindustriales entre dos países a partir del comercio de doble vía en bienes similares. Ha sido utilizado recientemente en diversas publicaciones relativas al comercio intraindustrial regional.

${ }^{27}$ El IGL es calculado a partir de la Clasificación Uniforme del Comercio Internacional (CUCI revisión 4 a 3 y 4 dígitos) tanto para los intercambios de los miembros de la AP entre sí como para el comercio realizado con sus tres principales socios asiáticos, para los años 2008, 2013 y 2014, siguiendo la metodología de Durán y Álvarez (2008). El IGL adquiere valores comprendidos entre 0 y 1 y se plantea que niveles inferiores a 0.1 señalan que existe comercio interindustrial entre dos países mientras que valores superiores o iguales a 0.33 indican la presencia de comercio intraindustrial (CI), y valores intermedios (superiores o iguales a $0.10 \mathrm{e}$ inferiores a 0.33 ) tienden a indicar comercio intraindustrial potencial (CIP).
La Alianza del Pacífico a ocho años de su creación. Balance crítico y perspectivas

Geneviève Marchini 


\begin{abstract}
Dossier
América Latina:

continuidad y cambio en el escenario regional
\end{abstract}

Existen también sectores con comercio intraindustrial entre los miembros de la AP y sus socios asiáticos, pero la situación de los tres países andinos difiere sensiblemente de la de México. Para los primeros, las relaciones intraindustriales son escasas y conciernen una muy baja proporción del intercambio bilateral; de hecho, el comercio bilateral agregado es de tipo interindustrial (valor del IGL < 0.10). En contraste, en el caso de México, las conexiones son más densas, incluyen un mayor número de sectores y una proporción más elevada del intercambio de bienes, en particular en las industrias automotriz y electrónica. Entre 2008 y 2017, el valor del IGL agregado se ha elevado e indica la presencia de comercio intraindustrial potencial en el par México-Japón en 2017. México podría, por ende, ser clave en el fortalecimiento de relacionamiento intraindustrial AP-Asia.

\begin{tabular}{|l|l|l|l|l|}
\hline \multicolumn{5}{|c|}{2008} \\
\hline & Chile & Colombia & México & Perú \\
\hline Chile & & & & \\
\hline Colombia & 0.15695717 & & & \\
\hline México & 0.15013608 & 0.14148975 & & \\
\hline Perú & 0.21429307 & 0.23393925 & 0.06497369 & \\
\hline
\end{tabular}

\begin{tabular}{|l|l|l|r|}
\hline \multicolumn{4}{|c|}{2008} \\
\hline & \multicolumn{1}{|c|}{ hina } & Corea & \multicolumn{1}{|l|}{ Japón } \\
\hline Chile & 0.02554172 & 0.00514315 & 0.00764937 \\
\hline Colombia & 0.02092253 & 0.00924807 & 0.0065232 \\
\hline México & 0.04798451 & 0.02545142 & 0.09322892 \\
\hline Perú & 0.00838395 & 0.00457528 & 0.00353713 \\
\hline
\end{tabular}

Tabla 1.

Alianza del Pacífico, índice de Grübel-Lloyd, intraAlianza y Alianza-Asia, 2008 y 2017

\begin{tabular}{|l|r|r|l|l|}
\hline \multicolumn{5}{|c|}{2017} \\
\hline & Chile & Colombia & México & Perú \\
\hline Chile & & & & \\
\hline Colombia & 0.14363889 & & & \\
\hline México & 0.17029653 & 0.24556382 & & \\
\hline Perú & 0.393044 & 0.2503802 & 0.12737392 & \\
\hline
\end{tabular}

\begin{tabular}{|l|l|l|r|}
\hline \multicolumn{5}{|c|}{2017} \\
\hline & \multicolumn{1}{|c|}{ China } & \multicolumn{1}{|l|}{ lorea } & Japón \\
\hline Chile & 0.01652819 & 0.00940572 & 0.00591128 \\
\hline Colombia & 0.00963517 & 0.02163524 & 0.01102744 \\
\hline México & 0.07642459 & 0.07063015 & 0.14516755 \\
\hline Perú & 0.00647228 & 0.02481404 & 0.0182656 \\
\hline
\end{tabular}

Fuente: autor con datos de COMTRADE. El IGL ha sido calculado a partir de la Clasificación Uniforme del Comercio Internacional (CUCI rev. 4 a 3 dígitos) siguiendo la metodología de Durán \& Álvarez (2008).

Ahora, ¿qué tan factible es alcanzar las metas de integración productiva intra-AP y entre la Alianza y Asia? Varias contribuciones señalan la dificultad del ejercicio. Las bases microeconómicas intra-AP no son sólidas (Torres 2016; Ramírez, León 2014) y la debilidad de estos vínculos reduce el atractivo de la Alianza. Villareal plantea que "los países tendrán probablemente que hacer esfuerzos considerables e involucrar fuertemente al sector privado para crear cadenas de suministro. Tendrán que enfocarse en las políticas adecuadas para crear las condiciones de su desarrollo en la región" (Villareal 2016: 13). Además de las inversiones ya señaladas, una política industrial regional es imprescindible para numerosos analistas (Vieira 2015; Ramírez, León 2013; Ghymers 2016; Marchini 2015). Es necesario ir más allá de la sola dinámica de la oferta y la demanda, y para lograr sus objetivos, "los países miembros deben retomar el rol regulador del Estado y adoptar políticas más adecuadas a los tiempos que vive la economía mundial" (Ramírez, León 2014: 4).

Ello nos lleva directamente a considerar el rol de las instituciones, otro aspecto ampliamente debatido. Si algunas revistas consideran que la institucionalidad ligera de la AP es un "mérito" y una "buena noticia para los inversionistas" 
(Kuczynski 2015, McKeigue 2013), el punto de vista prevaleciente en los análisis académicos es que la ausencia de instituciones supranacionales constituye una limitante o incluso un impedimento para que la AP logre sus objetivos más ambiciosos de libre circulación y creación de cadenas productivas regionales, en suma, la integración profunda que se plantea. La libre circulación de personas y capitales requiere "entes supranacionales decisorios que impongan patrones de conducta y administren el espacio económico. De lo contrario, se condenaría la eficacia del proceso a la revalidación política permanente de los estados miembros..." (Tremolada 2016: 80). En estas condiciones, se requeriría mantener el nivel de compromiso en la Alianza a lo largo de los cambios de gobierno en los cuatro países, (Naím 2014, Rodríguez 2015), una exigencia que tiende a fragilizarla. La institucionalización se plantea entonces como un paso necesario (Echebarría, Estevadeordal 2014: 41; Malamud 2012; Prado, Velázquez 2016: 208; Torres 2016: 24). "La capacidad de la AP para conseguir una integración profunda entre sus miembros pasa necesariamente por una estructura institucional que le permita atender este propósito" (Prado, Velázquez 2016: 208).

\section{La Alianza del Pacífico y la integración latinoamericana}

Finalmente, llegamos a una de las partes del debate tal vez más candente, la relación entre la AP y los demás mecanismos de integración latinoamericana y el impacto de la primera sobre el proceso regional. La amplitud de las opiniones vertidas es considerable: va desde contribuciones que consideran la AP como una iniciativa que agregaría dinamismo a América Latina (Naím 2014, Nolte 2016: 1), "un soplo de aire fresco en la región" (Heine, 2016), "un catalizador potencial para fortalecer la CELAC" (Ghymers 2016: 203) a aportaciones que plantean que la Alianza contribuye más a la fragmentación entre los latinoamericanos (Tremolada 2016: 75) o que la presentan como "la vía para intentar reencauzar a ALC en un proyecto de integración subordinado" (Regueiro 2014: 150). En esta sección, no abordaremos todos los aspectos de este debate, sino que nos centraremos en las contribuciones que discuten de los beneficios que aportaría una convergencia entre la AP y el MERCOSUR, vinculadas en particular con la CEPAL.

Estas aportaciones parten de la complementariedad de intereses económicos de las naciones latinoamericanas y de los beneficios que les pudiera significar acciones de cooperación en materia de integración económica regional y de relacionamiento con Asia. Se plantea que las acciones de cooperación con el MERCOSUR pueden elevar el atractivo de la Alianza. En efecto, se argumenta que el bajo nivel de interdependencia comercial en la AP y el tamaño insuficiente de su mercado interno reducen su potencial frente a posibles inversionistas, en especial asiáticos (Bárcena 2016, Ghymers 2016). Sería más interesante para la AP "promocionarse a sí misma como una plataforma privilegiada desde la cual hacer negocios con el resto de Latinoamérica" (Bárcena 2016: 62). El interés estratégico de la AP consistiría en "desarrollar una integración más
La Alianza del Pacífico a ocho años de su creación. Balance crítico y perspectivas Geneviève Marchini 
Dossier América Latina: continuidad y cambio en el escenario regional profunda con MERCOSUR sin renunciar a las ventajas de la apertura global" (Ghymers 2016: 237). Echebarría y Estevadeordal (2014) proponen que la AP deba considerarse "como una contribución a la integración de Latinoamérica abierta y no excluyente, que construya puentes entre proyectos subregionales [y] concebir su posición estratégica en el Pacífico como un activo para asegurarle a todos los países del continente una sólida proyección [...] hacia esta dinámica región" (Echebarría, Estevadeordal 2014: 41-42) y George afirma que tiene "el potencial para empujar América Latina hacia economías del estilo de los Tigres Asiáticos y un crecimiento de tipo Tigre Asiático" (George 2014: 30).

La creación y el fortalecimiento de cadenas de valor latinoamericanas es un elemento clave de estas propuestas. Ghymers señala que de hecho algunos miembros de la AP como Chile o Colombia están más integrados productiva y comercialmente con miembros del MERCOSUR que con los demás miembros de la Alianza (Ghymers 2016). Para Foxley, cabría “invertir la dirección del proceso de integración [...] en AL” hacia la integración desde la base productiva (Foxley 2014: 17). No hace falta subrayar que las condiciones que harían posibles este proceso son extremadamente exigentes: cero-aranceles intra-región, normas de facilitación del comercio, acumulación de origen, "fuertes inversiones conjuntas en energía, transporte, carreteras bioceánicas, puertos y aeropuertos [...] intercambio masivo de ingenieros y técnicos y de estudiantes [...] creación de un pool integrado de recursos humanos de alta calidad" (Foxley 2014: 19-20). La AP devendría "una 'Alianza del Pacífico Latinoamericana' orientada a la integración práctica y efectiva de los aparatos productivos desde el bottom up y no desde el top down", bajo el liderazgo regional de Brasil (Foxley 2014: 21). En un mismo tenor, Bárcena propone dos criterios adicionales para medir la contribución de la AP: cuanto puede contribuir a la integración latinoamericana y cuan efectiva puede ser como puente entre la región y Asia-Pacífico (Bárcena 2016: 64).

Por supuesto, no todos concuerdan con esta visión utópica. Así, Vieira plantea que la búsqueda de una "convergencia en la diferencia" perjudicaría el propio proceso de integración de la Alianza, al proponer distintos objetivos (Vieira 2015) y Ramírez y León (2013) se decantan por una primera etapa de continuidad geográfica a lo largo de las costas del Pacífico, retomando la configuración del Arco del Pacífico.

\section{Perspectivas en el nuevo entorno global}

Recientemente, la Alianza del Pacífico ha precisado sus metas en el documento "Visión Alianza 2030", donde los mandatarios de los países miembros establecieron cuatro ejes para alcanzar una Alianza "más integrada, más global, más conectada y más ciudadana”. De hecho, el documento estableció una meta precisa para el comercio intra-Alianza, y ha recalcado la vigencia del objetivo de fortalecimiento de las cadenas productivas regionales en la agenda de la AP. La primera meta ha sido confirmada en medio de una serie de obje- 
tivos generales de apertura, facilitación, fortalecimiento del sector financiero y del sector servicios: en el primer eje, se menciona de manera explícita la búsqueda de reducción de la dependencia de las materias primas ${ }^{28}$, y se plantea duplicar el comercio intra-Alianza a través de encadenamientos productivos y de la integración de las MPyMEs al comercio internacional.

Los instrumentos que deberán favorecer la internacionalización de las MPyMEs se precisan en el segundo eje, donde la consolidación de la integración regional se plantea como un aspecto de la "doble dimensión del modelo de integración de la AP”. Éstos son un mejor acceso al financiamiento, el apoyo a la difusión de sus productos, el apoyo al conocimiento y a las buenas prácticas fiscales y financieras. Asimismo, otras herramientas se mencionan en el tercer eje: la mejora de la infraestructura digital, la difusión de las TIC y la capacitación humana para conformar una economía digital regional. En suma, hasta la fecha, las políticas introducidas y propuestas para fortalecer los encadenamientos regionales son de tipo horizontal, destinadas a mejorar la competitividad general de todos los sectores productivos.

Es notable la ausencia de políticas sectoriales de fomento más específicas o de una política industrial conjunta; el informe 2018 de la UNCTAD señala que tres miembros de la AP establecieron políticas industriales en los últimos 5 años: Chile, Colombia y México, siendo Perú la única excepción a este patrón. Cabrá pues analizar el contenido y el alcance de estas políticas nacionales y su grado de congruencia y complementariedad entre sí y con los objetivos planteados por la agrupación. ¿Podría constituirse sobre esta base una política industrial de la Alianza que, dado el carácter intergubernamental del mecanismo, solo podría concebirse actualmente como una coordinación de políticas nacionales? Cabe señalar también que en el documento Visión 2030, no se mencionan compromisos concretos de los socios de la AP relativos a inversiones públicas, educación e Investigación y Desarrollo que permitirían sostener el fortalecimiento de los encadenamientos regionales. En el contexto internacional actual, donde la mayoría de las economías y en particular los socios asiáticos de la AP aplican políticas industriales más asertivas, cabe preguntarse si este conjunto de medidas será suficiente para impulsar una dinámica regional de fortalecimiento del comercio y de los encadenamientos productivos. ${ }^{29}$

Ello tanto más pues en la "Visión 2030" la AP, al reafirmar su compromiso con la globalización y el libre comercio, multiplica las iniciativas para avanzar en el segundo aspecto de la "doble dimensión" de su modelo de integración, el fortalecimiento de su red de acuerdos y tratados internacio-

28 El texto busca establecer en un plazo de doce años "un bloque comercial que crece de forma sostenible, dependerá menos de materias primas y contará con un mercado más competitivo, más eficiente, productivo y plenamente integrado". A nuestro juicio, se trata de una de las pocas novedades en esta lista de metas y objetivos que han ido apareciendo desde 2011.

29 El informe 2018 de la UNCTAD analiza la nueva tendencia global hacia la adopción de políticas industriales y señala que, entre 2013 y 2018, 84 países establecieron políticas industriales, entre éstos, los tres miembros de la AP ya citados.
La Alianza del Pacífico a ocho años de su creación. Balance crítico y perspectivas

Geneviève Marchini 
Dossier América Latina: continuidad y cambio en el escenario regional nales, que aparece mucho más detallado que las políticas de integración intra-Alianza. Estas comprenden tanto la articulación con foros internacionales (APEC, OCDE) y con otros bloques, como la ASEAN o la Unión Europea, como una mayor vinculación con países, a través del alza del número de Estados Asociados (10 en 2030) y del fortalecimiento de la cooperación con Estados Observadores. ${ }^{30}$ La impresión que prevalece es que el objetivo de apertura prima actualmente sobre el de integración regional, posiblemente debido al nuevo contexto global, más desafiante y ciertamente menos benigno para países que han apostado decididamente al mercado externo y poco al mercado interno. Así, como lo hemos visto, después de la elección de Donald Trump y la salida de Estados Unidos del TPP, la AP multiplicó las iniciativas - renovado diálogo con la ASEAN, acercamiento con el Mercosur, firma del nuevo TP-TPP por Chile, México y Perú. La creación de la categoría de países asociados y el inicio inmediato de negociaciones conjuntas con Australia, Canadá, Nueva Zelanda y Singapur constituyeron un cambio notable en el patrón anterior de negociaciones bilaterales. Estas iniciativas, tomadas en su conjunto, tienden a transformar la AP en un hub latinoamericano de TLCs y acuerdos comerciales.

Ahora bien, el impacto agregado de estas iniciativas es extremadamente difícil de predecir. Pueden favorecer el fortalecimiento o la creación de intercambios comerciales y de vínculos intraindustriales, a nivel de la subregión latinoamericana, como es el caso del acercamiento con MERCOSUR, con el cual ya existe un mayor nivel de comercio intraindustrial que con Asia. El acercamiento con los principales socios asiáticos puede también resultar positivo para la integración intra-AP pues China, Corea del Sur y Japón han manifestado un marcado interés por la construcción de infraestructuras, ${ }^{31}$ la generación y el transporte de energía, en especial energía renovable. Las propuestas relativas al desarrollo de sectores específicos coincidieron alrededor de los sectores tradicionales vinculados con los recursos naturales ${ }^{32}$, pero in-

${ }^{30}$ La Alianza del Pacífico ha acordado priorizar su trabajo con los Estados Observadores en cuatro áreas temáticas: ciencia, tecnología e innovación, facilitación del comercio, MPyMEs y educación.

31 Sus propuestas abarcan las infraestructuras de transporte, con propuestas tanto de parte de Japón (México), como de Corea y China, con los proyectos de Ferrocarril de los dos océanos, o los dos proyectos de desarrollo rural y de infraestructura planeados en Colombia. Las propuestas técnicas van, a su vez, asociadas a préstamos o acuerdos para financiar dichos proyectos; así, China empieza a diversificar sus inversiones financieras en América Latina más allá de los miembros del Mercosur y de Cuba para fondear estos importantes proyectos de infraestructura. Japón ha firmado varios Memorandos de Entendimiento entre instituciones financieras y ha concedido un préstamo especifico al Banco Mexicano de Comercio Exterior, y Corea y Colombia hicieron una primera aportación a un fondo común de financiamiento de infraestructuras.

32 Estos incluyen la minería y las cadenas de suministro mineras, la agricultura y la I\&D agroindustrial, la industria forestal y la cooperación en manejo de bosques, la pesquería, o en la relación México-Japón, el sector petrolero. 
cluyen también sectores más novedosos o de mayor nivel tecnológico -hierro y acero, materiales de construcción, equipo de ingeniería (China-Colombia), productos para la defensa (China-Chile), MPyMEs de las industrias creativas, TIC (Corea-Chile), industria espacial y astronomía (Corea-Chile y MéxicoJapón), Parques de Ciencia y Tecnología, biotecnologías, nuevos materiales y TIC (Corea-Perú).

En suma, estas nuevas propuestas de cooperación podrían apuntalar la creación de la necesaria infraestructura física y digital planteada por la AP, con una importante condición, la de que sus miembros presenten y negocien una postura conjunta y propuestas comunes, algo que hasta la fecha no se ha concretado. Por otro lado, si bien hay pocas propuestas de parte de los tres socios asiáticos relativas a encadenamientos productivos, un planteamiento conjunto de parte de la AP podría utilizar el carácter atractivo del mercado formado por los países miembros para atraer inversiones más allá de los sectores de interés tradicional e incluso fortalecer el desarrollo de actividades y de encadenamientos productivos de mayor nivel tecnológico.

Sin embargo, la apertura a una mayor competencia desde nuevos países, en particular Malasia o Vietnam a través del CP-TPP, no será necesariamente favorable o incluso puede contrarrestar el fortalecimiento del comercio y el comercio intraindustrial intra-AP. Por un lado, porque puede llegar a exponer en un plazo relativamente corto los productores regionales y en especial las MPyMES a importaciones de países más competitivos y/o con costos de producción menores en sectores importantes para el comercio intraindustrial intra-Alianza. Por otro, porque las necesarias inversiones en infraestructura para conectar el mercado interno de la AP y reducir los costos de transporte y comunicación tomarán tiempo para materializarse. El riesgo es, pues, que los miembros de la AP, sigan relacionándose con el mundo y en especial con Asia, sobre la base de su especialización actual, primaria-exportadora en el caso de los países andinos, plataforma de ensamblaje final de manufacturas para México, y que se debiliten los encadenamientos regionales que constituyen uno de los objetivos de la Alianza.

\section{Conclusiones}

En sus ocho años de existencia, la Alianza del Pacífico ha mostrado un gran dinamismo en sus iniciativas, las que han ido diseñando un mecanismo de integración con características originales que recoge explícitamente objetivos asociados a etapas anteriores del regionalismo latinoamericano -integración productiva, liberalización de flujos económicos entre los miembros, cooperación en distintos ámbitos- y pretende combinarlos con una agenda de apertura al mundo y en especial al Asia-Pacífico que pasa por el fortalecimiento de una red de acuerdos comerciales pero también por nuevas iniciativas de cooperación con los países observadores y asociados. Ahora bien, numero-
La Alianza del Pacífico a ocho años de su creación. Balance crítico y perspectivas Geneviève Marchini 
Dossier América Latina: continuidad y cambio en el escenario regional sos retos esperan el nuevo mecanismo. Algunos se relacionan con la evolución del contexto internacional, mientras otros son de carácter interno a la AP.

El primer reto de la Alianza proviene de su propio diseño y consiste en crear sinergias y avanzar simultáneamente hacia su doble objetivo. Ello significa, en primer lugar, elevar la interdependencia entre socios y avanzar hacia sus beneficios esperados en términos de mayor productividad, fortalecimiento de encadenamientos productivos regionales, y mayor inclusión de MPyMEs en el comercio exterior. En segundo lugar, debe progresar al mismo tiempo la integración internacional de los miembros de la AP, en especial con Asia, de una manera que permita corregir las profundas asimetrías que caracterizan los intercambios actuales, es decir a través de un proceso que permita diversificar y agregar valor a sus exportaciones, reduciendo su carácter primario-exportador. Para lograr estos propósitos, la Alianza cuenta con fortalezas, entre éstas, el carácter atractivo que ha logrado desarrollar, el interés y la colaboración del sector empresarial de los propios países miembros, y las oportunidades de cooperación con su importante red de estados observadores. El Primer Foro de Cooperación de la Alianza del Pacífico con sus Estados Observadores, que tuvo lugar en Lima los días 23 y 24 de abril de 2019, y donde se presentó un documento con oferta y demanda de proyectos de cooperación de parte de la AP, es un paso importante para movilizar esta red a favor de los objetivos de desarrollo de sus miembros.

Existen, sin embargo, numerosas dudas y preguntas relativas a estos objetivos y acerca de cómo alcanzarlos en un contexto global en rápida transformación. Las cuestiones cruciales se refieren, en particular, a las instituciones y a la "visión común" que sustentan a la Alianza. Ciertamente, la AP ha mostrado un grado de flexibilidad y capacidad de adaptación, por ejemplo, con la creación de la categoría de países asociados y el emprendimiento de negociaciones conjuntas, o con la formulación del objetivo de encadenamientos productivos regionales. Pero es posible que los propios objetivos de la Alianza, con la llegada de nuevos gobiernos al poder en los países miembros y la evolución del contexto internacional, requieran un grado mayor de flexibilidad.

Surge en primer lugar una serie de preguntas acerca de los medios con los cuales la Alianza se propone alcanzar su doble objetivo. ¿Es posible alcanzarlo solo a través de las políticas horizontales de apoyo a los sectores productivos que se han diseñado hasta la actualidad y sin una política industrial acordada entre los miembros? ¿Es posible lograr los objetivos planteados con un nivel de inversión pública que sigue siendo bastante bajo? ¿Cuánto tiempo se requerirá para poner en funcionamiento el FIAP y hacer realidad unos primeros proyectos de infraestructura? En otras palabras, se plantea la cuestión de si los miembros de la AP están dispuestos a flexibilizar su postura para ir más allá del liberalismo que profesan e incorporar políticas más voluntaristas para mejorar la calidad de su inserción en la economía global. Ello implica, por un lado, que la institucionalidad de la Alianza evolucione y vaya más allá de los 
mecanismos intergubernamentales que la caracterizan para incorporar mecanismos supranacionales y, por otro, que se vuelva aún más pragmática en materia de políticas económicas. Ello significaría modificar dos de sus pilares, su carácter intergubernamental y su visión liberal.

Otros eventos podrían llevar la Alianza a modificar o adaptar su visión liberal, para seguir funcionando en beneficio de una mejor inserción internacional de sus miembros, o a estancarse como lo hicieron otros mecanismos regionales. Se trata, en primer lugar, de los cambios de gobierno que se producen en los países de la AP. Cabe preguntarse ¿qué pasaría con el mecanismo y su dinámica si los nuevos gobiernos que lleguen al poder en los países miembros se encontrasen menos comprometidos con, o se opusieran a esta visión? Un posible ejemplo de los riesgos para la AP ya se manifestó con la llegada al poder del nuevo gobierno colombiano encabezado por Iván Duque, que se rehusó a participar en la sexta ronda de negociaciones que se desarrolló en septiembre de 2018 en Auckland (Nueva Zelanda) con los cuatro futuros países asociados. Desde entonces, el proceso parece haber seguido, aunque poco ha trascendido en la prensa. ${ }^{33}$ Por otro lado, la postura del nuevo gobierno mexicano encabezado por Andrés Manuel López Obrador parece haberse aclarado, reafirmando el interés del país por la Alianza, a través de una declaración de Marcelo Ebrard, Secretario de Relaciones Exteriores, que la calificó de "columna vertebral de la política exterior de nuestro país hacia América Latina" (Secretaría de Relaciones Exteriores de México 2019). En todo caso, es deseable que la Alianza del Pacífico, y América Latina, en general, aprendan de la ASEAN, un mecanismo que reúne países cuyos gobiernos tienen posturas ideológicas muy diversas y que ha logrado niveles de cooperación elevados en numerosos ámbitos de interés común, creando una notable dinámica regional.

Finalmente, los cambios en el contexto global crean un importante nivel de incertidumbre para la Alianza, porque hemos entrado en un período de cuestionamiento de las bondades de un comercio internacional plenamente liberalizado. La ideología del libre comercio deja lugar a un pragmatismo que incorpora las aportaciones de otras escuelas de pensamiento y matiza las prescripciones de política comercial. ¿Se podrá adaptar la AP a estos cambios? Y, last but no least, la creciente rivalidad China-Estados Unidos y las intenciones manifiestas de este último país de "recuperar" el liderazgo perdido en América Latina ponen en delicada situación los miembros de la Alianza, para quienes China es el primer o segundo socio comercial. ¿Será posible mantener relaciones comerciales con todos? El artículo 32.10 del nuevo USMCA (United States-Mexico-Canada Agreement) que debería reemplazar el TLCAN de hecho imposibilita la firma de tratados de sus miembros con

${ }^{33}$ La página web de la Alianza del Pacífico no registra nuevos avances, pero la XXI Reunión del Consejo de Ministros de la Alianza Del Pacífico de mayo de 2019 menciona la revisión de los avances de estas negociaciones (Secretaría de Relaciones Exteriores de México 2019).
La Alianza del Pacífico a ocho años de su creación. Balance crítico y perspectivas Geneviève Marchini 
Dossier América Latina: continuidad y cambio en el escenario regional economías que no sean reconocidas como economías de mercado por el propio Estados Unidos y constituye una clara amenaza. En este contexto, no cabe duda de que una posición consensuada del mecanismo apoyará los miembros en su relación con el mundo.

\section{Referencias bibliográficas}

Alianza del Pacífico (2011a), Declaración de Lima, Alianza del Pacífico, Lima, disponible en: https://alianzapacifico.net/documentos-y-estudios/.

Alianza del Pacífico (2011b), Memorando de Entendimiento sobre la Plataforma de Cooperación del Pacífico, Alianza del Pacífico, Bogotá, disponible en: https://alianzapacifico.net/ documentos-y-estudios/.

Alianza del Pacífico (2012), Acuerdo Marco de la Alianza del Pacífico, Alianza del Pacífico, Chile, disponible en: https://alianzapacifico.net/documentos-y-estudios/.

Alianza del Pacífico (2014a), Declaración de Cartagena de Indias, Alianza del Pacífico, Cartagena de Indias, disponible en: https://alianzapacifico.net/documentos-y-estudios/.

Alianza del Pacífico (2014b), Declaración de Punta Mita, Alianza del Pacífico, Punta Mita, disponible en: https://alianzapacifico.net/documentos-y-estudios/.

Alianza del Pacífico (2014c), Protocolo Adicional del Acuerdo Marco, Alianza del Pacífico, disponible en: https://alianzapacifico.net/documentos-y-estudios/.

Alianza del Pacífico (2018), Visión 2030, Alianza del Pacífico, disponible en: https:// alianzapacifico.net/documentos-y-estudios/.

Ardila M. (2015), La Alianza del Pacífico y su importancia geoestratégica, "Pensamiento Propio", no 42, pp. 243-262.

Balassa B. (1964), Hacia una teoría de la integración económica, en: Integración de América Latina. Experiencias y perspectivas, M. Wioncek (ed.), Fondo de Cultura Económica, México.

Baldwin R. (2013), Global Supply Chains: Why They Emerged, Why They Matter, and Where They Are Going, en: Global Value Chains in a Changing World, D. K. Elms, P. Low (eds.), Fung Global Institute, Nanyang Technological University, World Trade Organization, WTO Publications, Geneva.

Barbosa M., Buitrago R. (s. f.), La razón de ser de la Alianza del Pacífico: ¿Integración Profunda o Integración Estratégica?

Bárcena A. (2016), The Pacific Alliance: Trade, Investment and Strategic Challenges, en: Emerging Markets. The Pacific Alliance. Perspectives and opportunities for Latin America, M. Torres, J. Violante (eds.), European Institute of International Studies, Salamanca, pp. 45-64.

Bello (2015), How Deep is Their Love?, “The Economist”, 12 de marzo, disponible en: https:// www.economist.com/the-americas/2015/03/12/how-deep-is-their-love.

Blanco A. (2015), La Alianza del Pacífico: Un largo camino por recorrer hacia la integración, Wilson Center, Latin American Program, 1-20, enero.

Briceño J. (2012), La Alianza del Pacífico: la viabilidad de un naciente bloque regional, en: El Pacífico latinoamericano y su inserción internacional, M. Ardila (ed.), Pontificia Universidad Javeriana, Bogotá, pp. 139-142. 
Briceño J. (2014), Regional Dynamics and External Influences in the Discussions about the Model of Economic Integration in Latin America, "EUI Working Papers RSCAS" 2014(11), European University Institute, San Domenico di Fiesole.

Briceño J., Quintero M., Ruiz D. (2013), El pensamiento estructuralista de la CEPAL sobre el desarrollo y la integración latinoamericana: reflexiones sobre su vigencia actual, "Revista Aportes para la Integración Latinoamericana”, 19 (28) junio, pp. 1-34.

Brummer C. (2014), Minilateralism: How Trade Alliances, Soft Law, and Financial Engineering Are Redefining Economic Statecraft, Cambridge University Press, New York.

Bullón M., Cobarrubias F., Hernández G., Fuentes A. (2013), La Alianza del Pacífico, "Cuadernos de Nuestra América", 24(47), diciembre, pp. 79-108.

Cerqueria O. (2016), The Pacific Alliance: building discourses and realities, en: Emerging Markets. The Pacific Alliance. Perspectives \& opportunities for Latin America, M. Torres, J. Violante (eds.), European Institute of International Studies, Salamanca, pp. 173-183.

Dade C., Meacham C. (2013), The Pacific Alliance: An Example of Lessons Learned, "Center for Strategic \& International Studies", 1-11, julio.

Donnan S. (2015), Special Report. Pacific Alliance seeks leverage by joining bigger groupings, "Financial Times" 15 de septiembre, disponible en https://www.ft.com.

Durán J., Álvarez M. (2008), Indicadores de comercio exterior y política comercial: mediciones de posición y dinamismo comercial, Documentos de proyectos, CEPAL, Santiago de Chile.

Durán J., Cracau D. (2016, diciembre), The Pacific Alliance and its economic impact on regional trade investment. Evaluation and perspectives, "International Trade Series", 128, 5-53, diciembre, CEPAL, Santiago de Chile.

Echebarría L., Estevadeordal A. (2014), Alianza del Pacífico: Un Nuevo Motor de Integración Regional, en: Alianza del Pacífico: en el progreso de integración latinoamericana, A. Foxley, P. Meller (eds.), CIEPLAN \& Ukbar Ed., Santiago de Chile, pp. 27-42.

Foxley A. (2014), Nuevo desafío para América Latina: integración productiva, en: Alianza del Pacífico: en el proceso de integración latinoamericana, A. Foxley, P. Meller (eds.), CIEPLAN \& Ukbar Ed., Santiago de Chile, pp.13-26.

Foxley A., Meller P. (eds.), Alianza del Pacífico: en el proceso de integración latinoamericana, CIEPLAN \& Ukbar Ed., Santiago de Chile.

Garzón J. (2015), Latin American Regionalism in a Multipolar World, "Robert Schuman Centre for Advanced Studies" 23, 1-20, abril.

Garzón J., Nolte D. (2018), The new minilateralism in regional economic governance: Crossregionalism and the Pacific Alliance, en: Handbook of South American Governance, P. Riggirozzi, C. Wylde (eds.), Routledge, London/New York, pp. 173-189.

George S. (2014), The Pacific Pumas. An Emerging Model for Emerging Markets, Bertelsmann Foundation.

Ghymers C. (2016), The Pacific Alliance Potential catalyst for strengthening the CELAC and paving the way towards a genuine strategic alliance with the EU, en: Emerging Markets. The Pacific Alliance. Perspectives \& opportunities for Latin America, M. Torres, J. Violante (eds.), European Institute of International Studies, Salamanca, pp. 203-242.

Greene M., Arnson C. (2016), The Pacific Alliance: Ongoing Challenges to Trade Integration, "Wilson Center Latin American Program", 1-11, julio.
La Alianza del Pacífico a ocho años de su creación. Balance crítico y perspectivas

Geneviève Marchini 
Dossier América Latina: continuidad y cambio en el escenario regional
Grübel H., Lloyd P. (1975), Intra Industry Trade: The Theory and Measurement of International Trade with Differentiated Products, Macmillan, London.

Hernández A., Magnani B., Posadas C., Redondo J., Ruiz J., Dos Santos E. (2015), Sectores con mayor potencial para aprovechar la integración en la Alianza del Pacífico, "Observatorio Económico Latam, BBVA", BBVA, 30 de junio, disponible en: https://www.bbva.com/wpcontent/uploads/2016/06/Sectores-con-potencial-AP.pdf.

Heine J. (2016), Sigla nueva en sopa de letras: raíces y ramificaciones de la Alianza del Pacífico, "Revista Mexicana de Política Exterior", 106, enero-abril, pp. 35-48.

Heine J., Guoping W., Renfang L. (2016), China and the Pacific Alliance: from Market Sharing to Industrial Integration?, en: Emerging Markets. The Pacific Alliance. Perspectives \& opportunities for Latin America, M. Torres, J. Violante (eds.), European Institute of International Studies, Salamanca, pp. 243-263.

Jiménez L., López S. (2015), Integración de México a las dinámicas globales y la potencialidad de la Alianza del Pacífico, Universidad de Guadalajara, México.

Keppel S. (2013), Meet the Latin America's Serious Side: the Pacific Alliance, 28 de mayo, disponible en: https://abcnews.go.com/ABC_Univision/News/meet-latin-americas-sidepacific-alliance/story?id=19262599.

Kotschwar B. (2013), Will the Pacific Alliance Succeed in Latin America after Other Trade Pacts Have Failed?, Peterson Institute for International Economics, Wahington, DC.

Kuczynski M. (2015), Pacific Alliance Countries Draw Strength from Liberal Worldview, "Financial Times", 27 de septiembre, disponible en: https://www.ft.com

La República (2018), La otra reunión de la Alianza del Pacífico a la que el gobierno colombiano no asistió, 29 de agosto, disponible en: https://www.larepublica.co/economia/la-otrareunion-de-la-alianza-del-pacifico-a-la-que-el-gobierno-colombiano-no-asistio-2776191.

Leví M., Reggiardo G. (2016), La Alianza del Pacífico en el regionalismo sudamericano actual, "Revista Mexicana de Política Exterior", 106, enero-abril, pp. 187-204.

Lloyd P. (2008), Deep Integration in Regional Trading Agreements, en: Regional Trade Agreements in Asia, Tran Van Hoa, C. Harvie (eds), Edward Elgar, United Kingdom, pp. 16-28.

Malamud C. (2012), La Alianza del Pacífico: un revulsivo para la integración regional en América Latina, "Real Instituto Elcano. Ari”, 1-5, 27 de junio.

Malamud C. (2015), Regional Integration in Latin America: A Diagnosis of the Crisis, "Global Journey of Emerging Market Economies", mayo, pp. 199-207.

Maldonado G., Morales R., Schiavon J. (2016), Las bases sociales de la Alianza del Pacífico, "Revista Mexicana de Política Exterior", 106, enero-abril, pp. 133-164.

Marchini G. (2015), Flujos de Inversión Directa e Inserción en las Cadenas de Valor Globales: ¿Qué puede cambiar la Alianza del Pacífico?, en: Perspectivas y oportunidades de la Alianza del Pacífico, I. Rodríguez, E. Vieira (eds.), Editorial CESA, Bogotá, pp. 179-228.

Marchini G. (s. f.), La Alianza del Pacífico y Asia del Este: relaciones económicas, alcances del comercio intraindustrial y perspectivas de mayores encadenamientos productivos.

Marczak J., George S., Pérez M.F., Saldarriaga A. (2016), Pacific Alliance 2.0. Next steps in integration, "Atlantic Council", 1-17, mayo.

McKeigue J. (2012), A Lucrative Latin American Alliance, "Money Week", 1-7, 23 de agosto, disponible en https://moneyweek.com/. 
McKeigue J. (2013), The Pacific Alliance: a new Latin American Superpower, "Money Week", 1-6, 2 de abril, disponible en https://moneyweek.com/.

McKeigue J. (2013), What I Learned at a Colombian Country Club, "Money Week", 1-5, 28 de mayo, disponible en https://moneyweek.com/.

McKeigue J. (2014), This Is the Best Investment Play in Latin America, "Money Week", 1-5, 6 de mayo, disponible en https://moneyweek.com/

Naím M. (2009), Minilateralism: The Magic Number to Get Real International Action, "Foreign Policy", julio-agosto, disponible en: https://foreignpolicy.com

Naím M. (2014), The Most Important Alliance You've Never Heard of, "The Atlantic", 1-13, 17 de febrero, disponible en: https://www.theatlantic.com

Nolte D. (2016), The Pacific Alliance: Nation Branding through Regional Organisations, "GIGA Focus Latin America", 4 de agosto, pp. 1-13.

Pastrana E., Jost S. (eds.) (2016), Incidencias Regionales y Globales de la Alianza del Pacífico, Editorial Gedisa, S.A., Barcelona.

Pastrana E., Castro R. (2017), La Alianza del Pacífico: un Eje Articulador del Interregionalismo pos-TPP, "Anuario de Integración", 14, Coordinadora Regional de Investigaciones Económicas y Sociales, pp. 71-94, disponible en: http://www.cries.org/wp-content/ uploads/2018/04/006-Pastrana.pdf.

Perry G. (2014), The Pacific Alliance: A Way Forward for Latin American Integration?, Center for Global Development Essays, versión electrónica disponible en: http://www. cgdev.org/publication/pacific-alliance-way-forward-latin-american-integration.

Prado J., Velázquez R. (2016), La Alianza del Pacífico: comercio y cooperación al servicio de la integración, "Revista Mexicana de Política Exterior", 106, enero-abril, pp. 205-235.

Preciado J. (2018), Introducción, en: Dimensiones, estrategias y alternativas de la integración autónoma para América Latina y el Caribe. Desafíos para el caso mexicano 2010-2015. Tomo I, J. Preciado, S. Guerra, J. Estay, C. Oliva, Universidad de Guadalajara, México, pp. 27-92.

Ralph O. (2018), Global Catastrophe Bond Market Size Climbs to a Record \$30bn, "Financial Times", 6 de septiembre, disponible en: https://www.ft.com/content/d62827b2-b1e0-11e8$99 \mathrm{ca}-68 \mathrm{cf} 89602132$.

Ramírez J. (2016), The Pacific Alliance in Asia-Pacific: Parallels and legal limitations to interregional cooperation. Shaping the Pacific Alliance, en: Emerging Markets. The Pacific Alliance. Perspectives \& opportunities for Latin America, M. Torres, J. Violante (eds.), European Institute of International Studies, Salamanca, pp. 155-172.

Ramírez J., León J. (2014), La Alianza del Pacífico. Alcances, competitividad e implicaciones para América Latina, "Friedrich Ebert Stiftung Mexico", no 5, septiembre, pp. 1-58.

Rathbone J.P. (2014), Pacific Alliance Takes Pragmatic Approach to Integration, "Financial Times", 1 de abril, disponible en: https://www.ft.com.

Rathbone J.P. (2015), Pacific Alliance: Like Minds Aim to Tread United Line, "Financial Times", 27 de septiembre, disponible en https://www.ft.com.

Ravenhill J. (2011), Global Political Economy, Oxford.

Regueiro L. (2014), La Alianza del Pacífico: un pilar para el apuntalamiento del liderazgo global de Estados Unidos, "Revista de Estudios Estratégicos", no 1, enero, pp. 149-175.

Roberts J. (2014), The Pacific Alliance: A Latin American Role Model for the United States, The Heritage Foundation, 16 de mayo.
La Alianza del Pacífico a ocho años de su creación. Balance crítico y perspectivas

Geneviève Marchini 
Dossier América Latina: continuidad y cambio en el escenario regional
Rocha A., Morales E. (2015), Geopolítica de la Alianza del Pacífico en América Latina, el Continente Americano y Asia Pacífico, en: Perspectivas y oportunidades de la Alianza del Pacífico, I. Rodríguez, E. Vieira (eds.), Editorial CESA, Bogotá, pp. 105-152).

Rocha A. (2018a), Introducción. La dimensión político-institucional de la integración regional y subregional de la región (2000-2015), en: Dimensiones, estrategias y alternativas de la integración autónoma para América Latina y el Caribe. Desafíos para el caso mexicano 2010-2015. Tomo II., J. Preciado, A. Rocha, M. López, H. Cairo, Universidad de Guadalajara. México, pp. 35-40.

Rocha A. (2018 b), La CELAC en el proceso de construcción de una región autónoma global, en: Dimensiones, estrategias y alternativas de la integración autónoma para América Latina y el Caribe. Desafíos para el caso mexicano 2010-2015. Tomo II., J. Preciado, A. Rocha, M. López, H. Cairo, Universidad de Guadalajara. México, pp. 41-60.

Rodríguez I., Vieira E. (eds.) (2015), Perspectivas y oportunidades de la Alianza del Pacífico, Dodać: CESA, Bogotá.

Rodríguez I. (2015), Oportunidades y Desafíos que Plantea la Alianza del Pacífico para la Política Exterior y para los Nuevos Modelos de Integración Regional de sus Miembros, en: Perspectivas y oportunidades de la Alianza del Pacifico, I. Rodríguez, E. Vieira (eds.), Editorial CESA, Bogotá, pp. 33-62.

Roldán A., Castro A., Eusse M. (2013), Comercio intraindustrial entre las economías de Asia y el Pacífico y la Alianza del Pacífico, 2007-2011, CEPAL.

Saltalamacchia N., Urzúa M. (2016), Alianza del Pacífico: mitos y realidades, en: Incidencias Regionales y Globales de la Alianza del Pacífico, E. Pastrana, S. Jost (eds.), Fundación Konrad Adenauer, A.C, México, pp. 101-124.

Schiff M., Winters L. (2003), Regional Integration and Development, The International Bank of Reconstruction and Development, Washington.

Secretaría de Relaciones Exteriores de México (2019), Se llevó a cabo la XXI Reunión del Consejo de Ministros de la Alianza Del Pacífico, blog, 10 de mayo, disponible en: https:// www.gob.mx/sre/articulos/se-llevo-a-cabo-la-xxi-reunion-del-consejo-de-ministros-dela-alianza-del-pacifico-199677?idiom=es.

Senado de la República (2014), La Alianza del Pacífico ¿El futuro de la integración latinoamericana?, Centro de Estudios Internacionales Gilberto Bosques, Senado de la República, México.

Talvi E. (2014), Latin America's Decade of Development-less Growth, Think Tank 20: Growth, Convergence and Income Distribution: The Road from the Brisbane G-20 Summit, pp. 35-45.

The Economist (2013), A Continental Divide, 18 de mayo, disponible en: https://www. economist.com/the-americas/2013/05/18/a-continental-divide.

Tinbergen J. (1954), International Economic Integration, Elsevier.

Torres M. (2016), The New Model of Regional Integration for Emerging Countries in Latin America: The Pacific Alliance, en: Emerging Markets. The Pacific Alliance. Perspectives \& Opportunities for Latin America, M. Torres, J. Violante (eds.), European Institute of International Studies, Salamanca, pp. 19-44.

Tremolada E. (2016), Alianza del Pacífico: ¿una quimera?, en: Incidencias Regionales y Globales de la Alianza del Pacífico, E. Pastrana, S. Jost (eds.), Fundación Konrad Adenauer, A.C., México, pp. 71-100. 
UNCTAD (2018), Informe sobre las inversiones en el mundo. La inversión y las nuevas políticas industriales, UNCTAD, disponible en: https://unctad.org/es/PublicationsLibrary/ wir2018_overview_es.pdf.

Vásquez I. (2018), La Alianza del Pacífico: ¿Un retorno al modelo de regionalismo abierto en un contexto post-hegemónico? "Observatorio Colombiano de Tratados Comerciales", $15-18$, febrero.

Vieira E. (2015), La Alianza del Pacífico, ¿integración profunda hasta qué grado?, en: Perspectivas y oportunidades de la Alianza del Pacífico, I. Rodríguez, E. Vieira (eds.), Editorial CESA, Bogotá, pp. 63-104.

Villarreal A. (2016), The Pacific Alliance: A Trade Integration Initiative in Latin America, Congressional Research Service, 1-14, March.

Young A. (2017), The Politics of Deep Integration, "Cambridge Review of International Affairs", 30 (5-6), pp. 453-463.
La Alianza del Pacífico a ocho años de su creación. Balance crítico y perspectivas

Geneviève Marchini 